\title{
Modelling of TAE mode excitation with an antenna in realistic X-point geometry
}

\author{
A. Dvornova ${ }^{1,2,3}$, G.T.A. Huijsmans ${ }^{2,3}$, S. Sharapov ${ }^{4}$, F.J. Artola Such $^{5}$, \\ P. Puglia ${ }^{6}$, M. Hoelzl ${ }^{7}$, S. Pamela ${ }^{4}$, A. Fasoli ${ }^{6}$, D. Testa ${ }^{6}$ \\ 1 Aix-Marseille Universite, CNRS, PIIM UMR 7345, 13397 Marseille, France \\ 2 Eindhoven University of Technology, 5612 AZ Eindhoven, The Netherlands \\ ${ }^{3}$ CEA, IRFM, F-13108 Saint-Paul-lez-Durance, France \\ ${ }^{4}$ CCFE, Culham Science Centre, OX14 3DB, Abingdon, UK \\ ${ }^{5}$ ITER Organization, Route de Vinon sur Verdon, 13067 St Paul Lez Durance Cedex, France \\ ${ }^{6}$ EPFL, SPC, CH-1015 Lausanne, Switzerland \\ 7 Max-Planck-Institut fur Plasmaphysik, 85748 Garching, Germany \\ Thursday $9^{\text {th }}$ January, 2020
}

\begin{abstract}
Experimentally it is observed that TAE modes are difficult to excite with an external antenna when the plasma is in x-point geometry. Here, the effect of the X-point geometry on the efficiency of the TAE excitation with the external antenna is investigated. In the first part of the paper the influence of the near-LCFS (Last Closed Flux Surface) layer from the core side on the damping of the TAE modes is calculated using the linear resistive eigenvalue MHD code CASTOR. The resistive damping is identified as the main cause of the TAE damping in the open gap in the Alfven continuum. It is shown that one aspect of the difficulty of excitation of the TAE modes in X-point geometry is an increased damping from the region inside the separatrix. However, the increased damping with the plasma boundary approaching the separatrix is not general, and depends on the density profile shape. The second part of the paper discusses the influence of the TAE behaviour in the limiter and X-point geometries including the scrape-off layer (SOL) in the reduced MHD code JOREK. It is shown that the dominant effect on the damping of the original TAE mode observed in the limiter configuration is caused by the additional damping in the region of open field lines, i.e. the SOL.
\end{abstract}

\section{Introduction}

Fusion plasmas are characterized by the presence of high energy particles produced as a result of the fusion reactions, or generated by Ion Cyclotron Resonance, and neutral beam heating. The high performance of fusion devices requires the confinement time of these particles to be long enough for them to be thermalized and transfer energy to the bulk plasma before escaping the 
core region. A number of issues related to the redistribution and loss of these suprathermal particles was discovered. In tokamaks, one of the issues is the excitation of global shear Alfvén modes with their discrete eigenfrequencies existing in the gaps of the Alfvén continuum. These modes bear a generic name of gap modes. They can be driven by the free energy contained in the pressure gradient of the fast particles via wave-particle interactions since their velocity can be comparable with the Alfvén velocity: $v \sim v_{\text {Alfvén }}=B_{\text {tor }} / \sqrt{\mu_{0} \rho}$, where $B_{\text {tor }}$ is the toroidal magnetic field, and $\rho$ is the plasma mass density. Toroidal Alfvén Eigenmodes (TAEs) [1] is the name of modes located in the gap of continuum occurring due to the toroidicity which causes the coupling of $m$ and $m+1$ poloidal harmonics. Being a global mode, TAEs can lead to fast particles redistribution in space at nearly constant energy, and therefore affect fast particle confinement, reduce heating and current drive efficiency, cause damage to the first wall, and decrease overall plasma performance. Therefore, a clear understanding of the Alfvén modes dynamics is required for a careful operation of a fusion reactor.

TAE studies in the presence of fast particles are complicated due to the fact that the fast particle drive of the mode is changing since TAEs can cause redistribution of the particles. In order to exclude the effect of the altering particles drive, the dynamic of the TAE modes can be investigated by launching electromagnetic waves by an external antenna and detecting the TAE response in the form of resonance peaks with the saddle and pickup coils measuring perturbed radial and poloidal magnetic fields correspondingly with a synchronous detection technique.

The numerical results of the TAE excitation by an external antenna presented in this paper were motivated by the experimental observations on the JET tokamak, where excitation of TAE modes with an external antenna has been very successful [2]. There, the in-vessel saddle coils were used to drive an $\mathrm{n}=1$ perturbation as an excitation source for the TAEs. The driving current is typically $\sim 30 \mathrm{~A}$ and is chosen to be such that the magnetic field perturbations are of the order of $\delta B / B \sim 10^{-5}$, which is small enough to avoid magnetic configuration distortion and direct changes in the fast particle confinement.

In order to detect the TAE modes, the applied frequency of the antenna sweeps over the frequency position of the potential TAE gap until the mode is detected via magnetic diagnostics. As an initial guess for a TAE frequency, a rough estimate of $\omega_{T A E}=v_{A} / 2 q R$ can be made, where $q$ is the safety factor and $R$ is the major radius, and typically is $\sim 100-200 \mathrm{kHz}$. Once the mode has been detected, frequency sweeps are reduced from the range which allows to cover the whole toroidicity-induced gap to a range following the TAE resonance full width. An example of the frequency sweep range transition can be seen in the top figure in fig. 1 at $t=58 \mathrm{~s}$ for discharge \#42870. Knowing the response over the full resonance width, the resonance frequency and the damping rates determined as HWHM (half width at half maximum) of the resonant peak can be calculated (fig. 1(bottom)). A more detailed overview of the JET antennas used for the TAE excitation in the series of experiments used in this paper can be found in [3] and references therein. 
The analysis of the detected TAE peaks is based on a fitting of the transfer function presented in detail in [4]. The transfer function describes the amplitude of the output signal as a function of the frequency of the input signal:

$$
H(i \omega)=\frac{Y(i \omega)}{X(i \omega)}=\frac{F\{y(t)\}}{F\{x(t)\}},
$$

where $H(j \omega)$ is a transfer function, $y(t)$ and $x(t)$ are output and input signals, $F\{y(t)\}$ and $F\{x(t)\}$ denote the Fourier transform of the signals. In order to derive the transfer function for TAE detection, one simply needs to divide the output signal from diagnostics (such as pick up coils) by the input antenna current.

Presence of the Alfvén eigenmodes means existence of the poles in the derived transfer function, so that it can be represented as:

$$
H(j \omega)=\sum_{k=1}^{N} \frac{1}{i \omega-p_{k}},
$$

where $\mathrm{N}$ is a total number of resonances (corresponding to the total number of the TAEs in one gap), $\omega$ is the frequency of the input signal, and $p_{k}=\gamma_{k}+i \omega_{0 k}$ is a pole describing the $k$-th resonance. Fitting the transfer function to all the available signals provides the values for $\omega_{0}$ - the TAE peak position, and $\gamma-$ its damping rate in the absence of the fast particle drive. An example of such fit for discharge \#42870 is shown in fig. 2, where orange lines correspond to the fitted function and blue lines correspond to the raw signal.

However, while TAEs excited with an antenna were clearly visible in the limiter phase of the discharge, they disappeared when the X-point formed in the magnetic configuration, possibly due to an increase in the damping rates [5]. This effect can be seen in fig. 1. There, the TAE frequency of $150 \mathrm{kHz}$ and a damping rate $\gamma / \omega \approx 1.5 \%$ can be detected in the earlier stage of the discharge, and disappear after the transition to an X-point configuration occurring at $t \approx$ 51.3s. Similarly, the TAE can again be detected after the transition back to the limiter configuration around $t \approx 58.2 \mathrm{~s}$. It has to be clarified that the TAEs exist in toroidal plasmas, independent of whether or not the driving source is present in the experiment, and the commonly used formulation of TAEs not existing simply means that the modes don't have a high enough amplitude to be detected by the magnetic diagnostics or cause a significant redistribution of particles.

The previous extensive studies (e.g. [6], [7], [8], [9], and references therein) were performed to better understand the dynamics and damping mechanisms of global AE modes, showing that the damping rates increase significantly with shaping of the plasma and the gap between plasma and the external antenna, strongly depend on the variation of the plasma profiles at the edge, and are independent of the toroidal rotation shear.

The aim of the present work is to investigate in detail the effect of the Xpoint geometry on the efficiency of the TAE excitation with the external antenna 
and related damping rate. In this first study the simplest visco-resistive fluid MHD model is assumed to suffice. It is clear that a further study should aim to include more possible (kinetic) damping mechanisms [6]. However, this is left for future work. In the first part of the paper the influence of the near-LCFS (Last Closed Flux Surface) layer from the core side on the damping of the TAE modes is investigated using the linear resistive MHD code CASTOR [10]. The second part of the paper discusses the influence of the TAE behaviour in the limiter and X-point geometries including the scrape-off layer (SOL) in the nonlinear reduced MHD code JOREK [11]. The code allows to fully represent not only the core of the plasma, but the realistic geometry including the wall, the antenna, and the X-point with the SOL. The effects of the distance between the antenna and the width of the SOL on the TAE resonance behaviour were studied.

\section{Dependence of the damping rate on the near- LCFS layer}

Code description In the first part of the paper the behaviour of the TAE in proximity of the separatrix with plasma's boundary approaching the separatrix from the core side is studied. Here, three codes are used in order to first reconstruct the equilibrium, then reconstruct the Alfvén continuum for a given equilibrium and density profile, and then calculate the plasma response to an external antenna signal. The electron density profile was measured in these discharges with the LIDAR diagnostics, while the ion mixture $n_{T}: n_{D}$ was measured by the relative intensity of $T_{\alpha}$ and $D_{\alpha}$ spectral lines of $\mathrm{T}$ and $\mathrm{D}$ atoms at the plasma edge, and also by Neutral Particle Analyzer and Active TAE diagnostics from the variation in $v_{A}$ [5]. The EFIT code [12] was used in order to first reconstruct the experimental equilibrium. Further, the CSCAS [13] code was used for calculation of the the radial structure of the $n=1$ Alfvén continuum determined for the given input density and equilibrium previously produced by EFIT. CSCAS is based on the model described in [14], [15]. In this model an approximation of the dependence of the radial velocity and the magnetic field to be singular and logarithmic in nature is used according to [16], [17]. This approximation is only correct for the ideal MHD model, therefore plasma resistivity is not considered, and the resulting ideal continuum can only be used as a tool for finding an approximate TAE frequency and the location of TAE gap. This is due to an effect known as the Alfvén paradox discussed in [18], where it is demonstrated that the resistive MHD continuum is not identical to the ideal MHD continuum even when $\eta \rightarrow 0$.

The analysis of the plasma response to the sweeping antenna signal was performed with the code CASTOR. The set of linearised resistive MHD equations solved by CASTOR is as follows: 


$$
\begin{aligned}
\frac{\partial \rho_{1}}{\partial t} & =-\nabla \cdot\left(\rho_{0} \mathbf{v}_{\mathbf{1}}\right) \\
\rho_{0} \frac{\partial \mathbf{v}_{\mathbf{1}}}{\partial t} & =-\nabla p_{1}+\left(\nabla \times \mathbf{B}_{\mathbf{0}}\right) \times\left(\nabla \times \mathbf{A}_{\mathbf{1}}\right)+\left(\nabla \times \nabla \times \mathbf{A}_{\mathbf{1}}\right) \times \mathbf{B}_{\mathbf{0}} \\
\rho_{0} \frac{\partial T_{1}}{\partial t} & =-\rho_{0} \mathbf{v}_{\mathbf{1}} \cdot \nabla T_{0}-(\Gamma-1) \rho_{0} T_{0} \nabla \cdot \mathbf{v}_{\mathbf{1}} \\
\frac{\partial \mathbf{A}_{\mathbf{1}}}{\partial t} & =\mathbf{v}_{\mathbf{1}} \times \mathbf{B}_{\mathbf{0}}-\eta \nabla \times \nabla \times \mathbf{A}_{\mathbf{1}},
\end{aligned}
$$

where $\rho, p, \mathbf{v}$ and $T$ are plasma mass density, pressure, fluid velocity and temperature, $\mathbf{B}$ and $\mathbf{A}$ are magnetic field and magnetic vector potential, $\eta$ is the resistivity, and $\Gamma$ is the specific heat ratio. The values with a subscript 0 denote the equilibrium quantities, and the ones with a subscript 1 denote the perturbations. Assuming that the variables evolve exponentially in time (as $\left.a(\mathbf{r}, t)=a(\mathbf{r}) e^{\lambda t}\right)$, this system can be rewritten in a form of an eigenvalue problem of the form:

$$
\mathbf{R} \cdot \mathbf{u}=\lambda \mathbf{S} \cdot \mathbf{u}
$$

where $\mathbf{R}$ and $\mathbf{S}$ are complex matrices, and $\mathbf{u}$ is a vector of variables. The version of CASTOR used in this work [19] includes the external antenna which works as a driving source of a given frequency $\omega_{d}$. The system of equations can be represented instead of an eigenvalue problem in the following form:

$$
\left(\mathbf{R}-i \omega_{d} \mathbf{S}\right) \cdot \mathbf{u}=\mathbf{a}_{\mathbf{d}}\left(\omega_{d}\right),
$$

where $\mathbf{a}_{\mathbf{d}}\left(\omega_{d}\right)$ is a driving term. This system provides a stationary state solution. With the drive frequency $\omega_{d}$ sweeping around the TAE frequency imitating the experiment, the radial structure, frequency, and damping rate of the TAE can be determined. In the code, the main plasma is surrounded by a vacuum and an ideally conducting wall. The antenna is situated inside the vacuum. In a resistive plasma, the boundary conditions for the tangential and normal components of the magnetic field are the so-called free-boundary conditions, i.e. continuity of the magnetic field across the plasma-vacuum interface. The continuity of the total pressure (kinetic and magnetic) implies that the pressure perturbation goes to zero at the boundary in a resistive plasma. The shape of the ideally conducting wall is taken to be the shape of the JET vacuum vessel.

Case setup The equilibrium produced by the EFIT code from JET discharge \#42870 discussed earlier was used in this work. For the analysis, two equilibria at $t=52.3 \mathrm{~s}$ and $t=54.8 \mathrm{~s}$ with the plasma in the $\mathrm{X}$-point configuration were analyzed. Here the plasma boundary is taken to be a closed field line flux surface approaching the separatrix. Both CASTOR and CSCAS can model shaped equilibria closely approaching the separatrix, but cannot take into account the actual X-point due to the choice of the straight field line flux surface coordinate system. 
The Alfvén continua for both equilibria taken at the times $t=52.3 \mathrm{~s}$ and $t=54.8 \mathrm{~s}$ were reconstructed using the CSCAS code, with the equilibria reconstructions corresponding to the X-point phase of the discharge at $t=52.4 \mathrm{~s}$ and $t=54.8 \mathrm{~s}$ are illustrated in fig. 3 . The toroidal rotation was not taken into account in the simulations as its effect is expected to be negligible in the purely Ohmic dicharge. The Alfvén continuum with the q and normalised density profiles for the equilibria at $t=54.8 \mathrm{~s}$ are illustrated in fig. 4 . The experimental electron density in this moment was equal to $5 \cdot 10^{19} \mathrm{~m}^{-3}$. It can be seen that the TAE gap is closed due to an overlap with a continuum branch near the boundary. This means that the antenna signal of any frequency across the TAE gap will experience a strong continuum absorption when the propagating signal's frequency equals the local Alfvén frequency when crossing the continuum lines.

In order to exclude the effect of the continuum absorption, the original experimental density was varied in order to make the gap open to exclude the crossings and, therefore, the strong damping. Here the equilibrium at $t=54.8 \mathrm{~s}$ will be taken as an example. The original raw and fitted experimental density profiles are illustrated on the left in the fig. 5 in green, together with the corresponding Alfvén continua on the right in the same figure also in green. The experimental density profile was modified, and the two modified density profiles (corresponding to blue and red lines in the same plot) were chosen such that only the outer part of the continuum was changed, varying the width of the open TAE gap. While varying density, the equilibrium is kept constant, meaning that the pressure profile doesn't change, and the change of the density profile leads to a compensating change of the temperature profile. The uncertainty in the measured data allows such an assumption of a density variation in the pedestal region. The resulting Alfvén continua are on the right in the fig. 5 in red and blue, and an open gap in the normalised frequency range of $\omega / \omega_{A} \approx 0.23-0.4$ in the case in red and in the range $\omega / \omega_{A} \approx 0.23-0.47$ in the blue case is now present.

The sensitivity of the plasma response to the edge density profile is seen in the fig. 6 where the plasma response as a function of the applied antenna frequency is demonstrated with the use of the CASTOR code. It can be seen in the figure that the response corresponding to the original density profile (solid line) doesn't allow to identify the TAE, whereas clear TAE peaks corresponding to two new density profiles are visible. According to [20], a possible effect of the TAE damping induced in the open continuum gap in the proximity of the continuum tips can be taking place. Therefore, in this work it was decided to study the sensitivity of the TAE peaks to the shape of the density in the edge. The two different modified density profiles with lower density in proximity of the separatrix were chosen, leading to the different widths of the gaps.

One of the important parameters in the CASTOR code used here which influences the TAE damping in the absence of the continuum damping ([16], $[21],[22],[23])$ is the plasma resistivity. It defines the resistive damping, and, therefore, the width of the TAE peak, as illustrated in fig. 7 . It can be seen that the damping approximately scales as $\approx \eta_{\text {norm }}^{1 / 2}$. For the following studies in this 
chapter, a realistic temperature-dependent Spitzer resistivity profile was used with the resistivity value of $\eta=2.6 \times 10^{-8} \Omega \mathrm{m}$, comparable to the experimental one of $\eta \approx 5 \times 10^{-8} \Omega \mathrm{m}$. A slightly lower value of the resistivity than the experimental one was chosen in order to avoid high damping. The plasma viscosity is not taken into account.

A study of the convergence of the damping rate/peak heights on the radial resolution of the grid (the number of points in the radial direction) was performed. As was discussed above, in ideal MHD sound and Alfvén waves both cause singularities at the radial positions where the antenna frequency coincides with the local continuum frequency. The radial width of this singularity is determined by the strength of the damping mechanisms in plasma, therefore, resistivity in CASTOR. Therefore, the grid resolution in the simulations should be high enough to resolve the width of the near-singularity. The dependence of the maximal absorbed power (height of the TAE peak) on the grid resolution is shown in fig. 8. Simulations presented hereafter are performed with a radial resolution of six hundred radial cubic finite elements that was sufficient in order to achieve a good convergence.

The goal of the presented work is to study the effect of the transition from the $\mathrm{X}$-point to limiter configuration on the TAE stability/damping rate. For this purpose, the original equilibria was "cut" along a certain flux surface so that there are only closed flux surfaces present in the modelling domain. This maximal outermost flux surface taken into account (which will be called $\psi_{\max }$ in this work) can be chosen arbitrarily. Therefore, by varying $\psi_{\max }=0.95, \ldots, 0.995$ with $\psi_{\max }=1$ corresponding to a separatrix, it is possible to evaluate the change of the TAE damping rate with plasma simulation boundary approaching the separatrix.

For a fixed $\psi_{\max }$ the simulation with the CASTOR code was performed by sweeping the frequency of the applied antenna signal, mimicking the experiment. As an example, let us consider an equilibrium at $t=54.8 \mathrm{~s}$ and the first density profile corresponding to the Alfvén continuum in fig. 5 in red. The sweep across the open gap around $0.25-0.4 \omega / \omega_{A}$ results in response functions corresponding to different fixed $\psi_{\max }$ are illustrated in fig. 9a). Two TAE response peaks can be identified: the first minor peak with a lower frequency at $f \approx 100 \mathrm{kHz}$, and the second main peak with a higher frequency at $f \approx 130 \mathrm{kHz}$. The corresponding damping rates of the two identified peaks calculated as HWHM are illustrated in fig. 9b). The damping rates of the first minor peak are not indicated for the $\psi_{\max }=0.99,0.995$ as the damping rates are so high that the peak is not distinguishable. The main resonance at $f \approx 130 \mathrm{kHz}$ shows a damping rate similar to the experimental values of $1-2.5 \%$ in this discharge. The damping rate increases by a factor of three with the boundary approaching the separatrix, i.e as $\psi_{\max } \rightarrow 1$. Mode structures for both TAEs at $t=54.8 \mathrm{~s}$ are illustrated in fig. 10 and fig. 11 respectively.

It is worth mentioning that the Alfvén continua discussed before did not include sound waves with frequencies $\omega_{S}=k_{\|} \sqrt{\Gamma p / \rho}$. The inclusion of them is illustrated in fig. 12 as a combination of Alfvén and sound continua in black in comparison with the same continua but only with inclusion of the Alfvén 
waves in red. This will result in the overlap of the sound continuum with the toroidicity-induced open Alfvén gap leading to the change of the damping rate due to the absorption of the antenna wave energy by the sound wave at the locations where the antenna frequency matches the local sound wave frequency.

Sound waves were included in all the simulations described in this chapter. Their inclusion can be noticed if looking closely at the mode structure of the parallel velocity, where sharp singularities correlate to the crossings of the sound continuum discussed above. In comparison, the mode structures corresponding to the same case but with $\Gamma$ set to zero is illustrated in fig. 13. To determine the influence of the damping due to the sound waves, a set of simulations for the main TAE peak at $f \approx 130 \mathrm{kHz}$ without the inclusion of the sound waves was performed by setting $\Gamma=0$. The results are demonstrated in the fig. 14 . It can be seen in the figure that even though the sound waves have an influence on the damping rates, the difference does not change the global trend of the damping rate increase with increase of $\psi_{\max }$. The influence of the sound waves increases with the value of the poloidal beta, which in this case is $\beta_{p}=0.74$, in agreement with results demonstrated in [19], [24]. It should be mentioned, that for a high $\beta_{p}$ values a more sophisticated kinetic treatment is required.

Similarly to the example above, for four cases total with both two equilibria and two density profiles the frequency sweeps across the TAE gap range were performed, and in each case two TAEs are identified. Only one TAE can be traced in the experiment, but it is likely that the dominant resonance with higher frequency is measured.

The damping rates for the first TAE with lower frequency and the second TAE peak with higher frequency for all four cases are shown in fig. 15 and in fig. 16 respectively. Overall, damping rates of $1-3 \%$ of the main resonance are consistent with the experimental observations for the same discharge. It can also be seen in these figures that damping rates for the first density are overall higher for both TAE peaks in both equilibria due to the fact that the first density is higher at the edge, which leads to lower temperatures and higher resistivities taking into account constant pressure. The effect is less pronounced for the lower frequency peak since it is localised closer to the core, and the change of resistivity in the edge does not influence it as much as the higher frequency peak. The sudden decrease in the damping rate for $\psi_{\max }$ increasing from 0.97 to 0.98 can be explained by the change of q profile: the maximal value of $\mathrm{q}$ changes from $q=4$ to $q=5$, therefore the mode structure and consequently frequency changes. As expected, the damping rate increases when approaching the separatrix. This result indicates that one aspect of the difficulty of excitation of the TAE modes in X-point geometry is an increased damping from the region inside the separatrix. However, the increased damping with the plasma boundary approaching the separatrix is not general, and depends on the density profile shape. 


\section{TAE excitation in $\mathrm{X}$-point geometry}

Section 2 was concentrating on the change of the TAE behaviour with the plasma boundary approaching the separatrix from the core side. In the second part of the paper the causes of the TAE damping are studied in the JOREK code in full X-point geometry.

Code description The simulations were performed using the non-linear MHD code JOREK. The MHD model used in this work is a single-fluid visco-resistive reduced MHD. The reduced model is deduced [25] from the standard MHD equations by substituting $\mathbf{B}=\mathbf{B}_{\phi}+\mathbf{B}_{\mathbf{p}}=F_{0} \nabla \phi+\nabla \psi \times \nabla \phi$ and $\mathbf{v}=\mathbf{v}_{\|}+\mathbf{v}_{\perp}=$ $v_{\| \mid} \mathbf{B}+R^{2} \nabla \phi \times \nabla u$, therefore assuming constant in time toroidal magnetic field. Here, $F_{0}=B_{0} R_{0}$ is the strength of the magnetic field at the geometric axis $R=R_{0}$, and $\phi$ is a toroidal angle. The resulting set of equations describes the evolution of the 7 variables, namely, $\rho$ - mass density, $T$ - temperature, $\psi$ poloidal magnetic flux, $v_{\|}$- parallel velocity, $u$ - electric potential, $j$ - toroidal current, and $w$ - vorticity:

$$
\begin{aligned}
\frac{\partial \rho}{\partial t} & =-\nabla \cdot(\rho \mathbf{v})+\nabla\left(D_{\perp} \nabla_{\perp} \rho\right)+\nabla^{2}\left(D_{\perp h y p} \nabla_{\perp}^{2} \rho\right) \\
\frac{\partial(\rho T)}{\partial t} & =-\mathbf{v} \cdot \nabla(\rho T)-(\gamma-1) \rho T \nabla \cdot \mathbf{v} \\
& +\nabla\left(\kappa_{\perp} \nabla_{\perp} T\right)+\nabla^{2} \cdot\left(\kappa_{\perp h y p} \nabla_{\perp}^{2} T\right) \\
\frac{\partial \psi}{\partial t} & =\eta j+R[\psi, u]-F_{0} \frac{\partial u}{\partial \phi} \\
\rho B^{2} \frac{\partial v_{\|}}{\partial t}+\rho v_{\|} \frac{\nabla \psi}{R^{2}} \frac{\partial \nabla \psi}{\partial t} & =-\rho|B|^{2}\left[u, v_{\|}\right]-\frac{1}{2} \rho v_{\|}\left[u, B^{2}\right]-\rho \frac{F_{0}}{2 R^{2}} \partial_{3}\left(v_{\|}^{2} B^{2}\right) \\
& +\frac{1}{2} \rho\left[\psi, v_{\|}^{2}|B|^{2}\right]-\frac{F_{0}}{R^{2}} \partial_{3} p-\frac{1}{R}[p, \psi] \\
R \nabla \cdot\left[R^{2} \rho \nabla_{\perp}\left(\frac{\partial u}{\partial t}\right)\right] & =\left[R^{4} \rho w, u\right]-\frac{1}{2}\left[R^{2} \rho, R^{2}\left|\nabla_{\perp} u\right|^{2}\right] \\
& -\left[R^{2}, p\right]+[\psi, j]-\frac{F_{0}}{R} \frac{\partial j}{\partial \psi}+\mu R \nabla^{2} w \\
w & =\nabla \cdot \nabla_{p o l} u \\
j & =\Delta^{*} \psi=R^{2} \nabla \cdot\left(\frac{1}{R^{2}} \nabla_{p o l} \psi\right) .
\end{aligned}
$$

Here, the $[A, B]=e_{\phi} \cdot \nabla A \times \nabla B$ notation is used to represent the Poisson bracket, and $\nabla_{p o l} A=\partial_{R} A \mathbf{e}^{\mathbf{R}}+\partial_{Z} A \mathbf{e}^{\mathbf{Z}}$. A temperature-dependent Spitzerlike resistivity has been used in the simulations: $\eta=\eta_{0}\left(T / T_{0}\right)^{-3 / 2}$, with $\eta_{0}=2.6 \times 10^{-9} \Omega \mathrm{m}$. The value of the resistivity was required to be lower than experimental in order to avoid high damping. The value of the viscosity was set to $4 \times 10^{-9} \mathrm{~kg} /(\mathrm{m} \cdot \mathrm{s}$, which was chosen such to facilitate the convergence of the simulations, and does not have a significant effect on the value of the 
resulting damping rate. $\mathrm{s}$, and does not have a significant effect on the value of the resulting damping rate. The hyper-diffusivities $D_{\perp \text { hyp }}=10^{-8} \mathrm{~m}^{4} / \mathrm{s}$ and $\kappa_{\perp \text { hyp }}=5 \times 10^{-15} \mathrm{~kg} \cdot \mathrm{m} / \mathrm{s}$ were used in the simulations to improve the numerical stability.

Cubic Bezier finite elements are used for the spatial discretisation in the poloidal plane, while a Fourier representation is used in the toroidal direction. The choice of the finite elements in poloidal plane allows a very flexible discretisation with the geometry including the accurate representation of the X-point. Finite elements are constructed in such a way that they are aligned with the flux surfaces of the initial equilibrium, which allows to accurately represent the fast parallel trans port in the SOL. The time discretisation uses the implicit Crank-Nicolson scheme such that the time step necessary for simulations is not restricted by the grid size. The simulations were performed with a time step of $\delta t=0.5 / \sqrt{\mu_{0} \rho_{0}} \mathrm{~s}$ which is sufficiently small to resolve the typical TAE frequencies (up to $200 \mathrm{kHz}$ ).

The JOREK code is coupled with the STARWALL code which allows to include active coils [26] enabling the simulation of the excitation of TAE modes with an external antenna in full X-point geometry, including the scrape-off layer. The JOREK domain includes the main plasma and the SOL. In the JOREKSTARWALL code, the JOREK domain is surrounded by a vacuum and a resistive wall. As for the CASTOR code, the antenna is situated in the vacuum, and the free-boundary conditions, i.e. continuity of the magnetic field, are used. The magnetic field boundary conditions are calculated by the STARWALL code once before a JOREK simulation for a given plasma and wall geometry. The resulting so-called response matrices are used in JOREK for the magnetic boundary conditions and the time evolution of induced currents in the resistive wall. Here the resistive wall is modelled on the JET vacuum vessel (see fig.21). The wall resistivity is taken to be $2.5 \times 10^{-1} \Omega / \mathrm{m}$. On the open field lines, leaving the JOREK domain, the boundary conditions on the density, temperature and parallel velocity are so-called divertor sheath boundary conditions: the parallel velocity is set to the local sound speed, the condition on the heat flux links the parallel convected and conducted energy through a sheath transmission factor. The density is flowing out naturally. The potential is set to zero on the boundary. On the parts of the JOREK boundary where it is aligned with the magnetic field, the conditions on the temperature, density, parallel velocity and electric potential at the boundary of the JOREK domain are such that the perturbations with respect to the initial state are set to zero on the JOREK boundary (i.e. outside the SOL).

Unlike CASTOR, the JOREK code was used in a time dependent mode. The plasma-vacuum-antenna system is evolved in time until a stationary state is obtained. The plasma behaves as a driven oscillator, and therefore the response function will look differently for a resonant and off-resonant cases. An example of such temporal evolution of the kinetic energy of $n=1$ mode for a limiter case is illustrated in fig. 17. Here, the dashed curve in grey corresponds to the resonant case with frequency $f=114 \mathrm{kHz}$, and the solid black curve corresponds to the off-resonant case with $f=120 \mathrm{kHz}$. In this example it can be seen that 
plasma behaves as a driven damped harmonic oscillator, with its non-resonant response initially growing, but eventually settling down to a steady pattern. Contrarily, its resonant response amplitude grows monotonically until it reaches its final value. The time needed for the steady solution to be reached is inversely proportional to the damping present in the system. The typical simulation time therefore depends on the chosen resistivity, and requires $\approx 10^{3}$ Alfvén times in this set of simulations.

Case setup In order to isolate a specific effect of the presence of the scrapeoff layer (SOL) the initial equilibrium taken from the JET shot \#42870 at the moment of time $t=54.88 \mathrm{~s}$ which corresponds to the $\mathrm{X}$-point phase of the discharge was analyzed in two ways. In the first case the plasma boundary was set at the flux surface $\psi_{\text {norm }}=0.995$, therefore not including the SOL, and in the second case the whole equilibrium with a real X-point and the SOL was taken. The examples of the grids for both cases are illustrated in fig. 18. The $\mathrm{n}=0$ mode of the equilibrium and initial profiles are kept constant throughout the simulation for both cases, only allowing excitation of the $n=1$ mode without a change of the Alfvén continuum. The density profile, and, therefore, Alfvén continuum correspond to the ones referred to as first modified density profile used previously in the studies with the CASTOR code shown in fig. 5 in red. The scans across the frequency range performed for both cases together with the corresponding CASTOR case are shown in fig. 19. In the no-SOL case (marked with squares in the figure), two TAE peaks are found which are split into a main resonance with a frequency $f_{1}=114 \mathrm{kHz}$ and a smaller peak just at the upper edge of the TAE gap with a frequency $f_{2}=136 \mathrm{kHz}$. Since in the no-SOL case the X-point geometry is not taken into account, the result can be compared to the one produced by the CASTOR code for the same equilibrium. The case analyzed with the CASTOR code is presented in the same figure in a line with star markers with the height of the peak normalized to the same value as the main resonance in the no-SOL JOREK case. The two peaks demonstrate good agreement with the main TAE peak in CASTOR having a slightly higher frequency of $f=125 \mathrm{kHz}$, and a lower damping rate of $\gamma / \omega=2.1 \%$ in CASTOR versus $\gamma / \omega=4.2 \%$ in JOREK. A direct comparison of continua in the two codes is not possible since JOREK is a time-dependent code, and the continuum for it cannot be calculated directly. As both codes are run using the same initial equilibrium and input profiles, and the $\mathrm{n}=0$ mode JOREK is not evolving, one can say with a significant confidence that the Alfven continua are identical at the beginning of the simulations. A possible explanation for the difference on the resonance frequency between the CASTOR and JOREK results is the presence of the viscosity terms in the JOREK model which are not included in the CASTOR MHD model. This may lead to a small modification of the frequency of the TAE modes. The peaks in both codes have a similar mode structure illustrated in fig. 20.

If in the no-SOL case the strong resonance observed both in the experiment and in the CASTOR code is recovered, it disappears in the case with the SOL 
and X-point included. The result of the simulation for this case with X-point is illustrated in fig. 19 in a line with circular markers. As can be seen, only one of the two original resonances remains, namely the secondary peak at $f_{2}=136 \mathrm{kHz}$.

The 2D poloidal mode structures for the no-SOL and SOL cases are shown in fig. 21. Note that the amplitude of the modes in the figure have different scales. In the case with no SOL included (top row), the first mode exhibits an even mode structure [27] with a maximum amplitude on the low field side, whereas the second mode with a higher frequency is an odd mode, and its amplitude is higher on the high-field side. Comparing the dominant modes in the no-SOL and SOL cases (on the left in the fig. 21, top and bottom respectively) it is clearly visible that the mode disappears, when the second mode with a higher frequency (on the right in the figure) remains.

As in the case with the CASTOR code, the necessary poloidal resolution has to be used in the simulations to properly resolve the thin resistive layer. The results of the simulations performed with a different poloidal resolution of the grid are shown in fig. 22, while the frequency of the antenna and its position were not changed. A resolution of 101 poloidal points was used in the simulations without the $\mathrm{X}$-point, while the $\mathrm{X}$-point cases required a more accurate grid with 151 points.

Antenna position The effect of the distance between the antenna and the plasma could influence the identification of the mode. A scan of the antenna position with regard to the plasma boundary, i.e. the last surface taken into account, was performed while keeping the antenna shape and frequency of the oscillation constant. Note that in both cases the antenna remains outside of the JOREK grid. The results of the scan for both SOL and no-SOL cases are illustrated in fig. 23. In both cases the amplitude of the mode has significantly increased (by more than $50 \%$ in the SOL case and by $\tilde{3} 0 \%$ in the no-SOL case) with antenna getting closer to the plasma boundary.

For two different antenna positions the frequency scans were performed, with results presented in fig. 24. As expected, the damping rate does not depend on the antenna position. However, moving of the antenna closer only influences the amplitude of the mode, therefore complicating the excitation and consecutive identification of the mode.

Influence of the SOL width A possible explanation of the increased damping in the X-point phase of the discharge is the existence of the SOL. A set of simulations with the SOL width being varied from $0 \mathrm{~cm}$ (corresponding to the case of no SOL present) to $2 \mathrm{~cm}$ was performed in attempt to follow the evolution of the TAE mode. Note that the SOL width here denotes the actual physical width of the SOL in the midplane taken into account in the simulations, not the e-folding length. In the fig. 25(left) the midplane temperature and density profiles are illustrated, with the close ups on profiles in the SOL region on the right of the figure.

The results of the SOL width scan are presented in fig. 26. In the figure, 
the initial response peaks of the kinetic energy of $n=1$ TAE mode with the SOL width $d_{S O L}=0 \mathrm{~cm}$ corresponding to the no-SOL case discussed above at $f=113 \mathrm{kHz}$ (main peak) and $f=137 \mathrm{kHz}$ (secondary peak) are shown in circular markers. While the secondary peak remains at its place with the increase of the SOL width, the main peak is experiencing a strong damping with its amplitude gradually decreasing with the increase of the SOL width. The related mode structures corresponding to the main peak are shown in fig. 27, with the mode amplitude slowly vanishing as the SOL width increases.

A possible explanation of the increased damping in the SOL is existence of an Alfvén continuum on the line-tied open field lines in analogy to what was proven to exist in [28] for coronal loops, which could cause the continuum damping of the mode in the SOL.

If the theory applies to the tokamak case, one would expect a sudden change in the damping rate with the increase of the SOL width once the resonant surface is included in the SOL. To check this theory, the damping rates can be calculated knowing the peak width as was done in the Sec. 2, and the results are shown in fig. 28(bottom) with the amplitude of the main peak evolution (top). In can be denoted from the figure that the damping increment is a smooth function, which disagrees with the suggestion of the existence of the continuum. Therefore, the main cause of the increased damping coming from the SOL region is believed to be a wave cut-off by the SOL with short fieldline lengths, but this assumption requires further investigation.

\section{Conclusions}

This paper discusses the effect of disappearance of the TAE mode peaks occurring with the transition from the limiter phase of the discharge to the X-point phase observed experimentally on JET with the saddle coils used as an external TAE antenna and made an attempt to identify the reasons causing this effect.

In the first part of the paper the influence of the near-SOL layer from the plasma core side with the use of the linear resistive MHD code CASTOR was studied. The results for two different magnetic configurations and two density profiles chosen such that the gap in Alfvén continuum is kept open to exclude the continuum damping were obtained. In both cases two TAE resonances located in the open gap were identified, with damping rates of up to $6 \%$ for the first minor peak with lower frequency and of $1-3 \%$ for a major peak with higher frequency, which are comparable values with the typical experimental results. The TAE with a lower frequency does not exhibit any specific trend with the domain boundary approaching the separatrix. However, the main TAE peak with the higher frequency in all four cases has a trend of the increased damping coming from the near-SOL region.

The second part of the paper was concentrated around a study of the presence of the separatrix and the SOL on the mode behaviour in the non-linear resistive MHD code JOREK coupled to the STARWALL code, allowing to directly

model the experiments with the sweeping antenna signal. Including X-point and 
SOL, the results similar to the ones obtained before in the experiment were recovered, namely the absence of the detectable mode in the X-point case. The influence of the distance between the plasma and the antenna and the damping in the SOL were addressed. The antenna positioning had affected the TAE amplitude with the move from $10 \mathrm{~cm}$ to $1 \mathrm{~cm}$ away from the boundary causing the mode amplitude increase by $30 \%$. It was shown that the dominant effect on the damping of the original mode observed in the limiter configuration is caused by the change of the SOL width.

This work has been carried out within the framework of the EUROfusion Consortium and has received funding from the Euratom research and training programme 2014-2018 and 2019-2020 under grant agreement No 633053. The views and opinions expressed herein do not necessarily reflect those of the European Commission. The authors are very grateful to $X$. Garbet, R. Dumont, M. Becoulet, and S. Pinches for useful discussions in preparing the manuscript of this paper.

\section{References}

[1] C. Cheng, L. Chen, and M. Chance, Annals of Physics 161, 21 (1985).

[2] A. Fasoli, D. Testa, S. Sharapov, H. L. Berk, B. Breizman, A. Gondhalekar, R. F. Heeter, M. Mantsinen, and contributors to the EFDA-JET Workprogramme, Plasma Physics and Controlled Fusion 44, B159 (2002).

[3] A. Fasoli, D. Borba, G. Bosia, D. J. Campbell, J. A. Dobbing, C. Gormezano, J. Jacquinot, P. Lavanchy, J. B. Lister, P. Marmillod, et al., Phys. Rev. Lett. 75, 645 (1995).

[4] J. Moret, CRPP Laboratory Report LRP 498, 94 (1994).

[5] A. Fasoli, D. Testa, T. Panis, A. Klein, J. Snipes, J. Sears, M. Gryaznevich, R. Martin, S. Pinches, et al., Plasma Physics and Controlled Fusion 52, 075015 (2010).

[6] A. Jaun, A. Fasoli, and W. W. Heidbrink, Physics of Plasmas 5, 2952 (1998).

[7] D. Testa, A. Fasoli, et al., Nuclear Fusion 41, 809 (2001).

[8] J. Snipes, N. Basse, C. Boswell, E. Edlund, A. Fasoli, N. Gorelenkov, R. Granetz, L. Lin, Y. Lin, R. Parker, et al., Physics of Plasmas 12, 056102 (2005).

[9] D. Testa, C. Boswell, and A. Fasoli, Nuclear fusion 45, 907 (2005).

[10] W. Kerner, J. Goedbloed, G. Huysmans, S. Poedts, and E. Schwarz, Journal of computational physics 142, 271 (1998).

[11] G. Huysmans and O. Czarny, Nuclear fusion 47, 659 (2007).

[12] D. O'Brien, L. Lao, E. Solano, M. Garribba, T. Taylor, J. Cordey, and J. Ellis, Nuclear fusion 32, 1351 (1992).

[13] S. Poedts and E. Schwartz, Journal of computational physics 105, 165 (1993).

[14] J. Goedbloed, The Physics of Fluids 18, 1258 (1975).

[15] Y.-P. Pao, Nuclear Fusion 15, 631 (1975).

[16] H. Grad, Physics Today 22, 34 (1969). 
[17] L. Chen and A. Hasegawa, The Physics of Fluids 17, 1399 (1974).

[18] D. Borba, K. S. Riedel, W. Kerner, G. T. A. Huysmans, M. Ottaviani, and P. J. Schmid, Physics of Plasmas 1, 3151 (1994).

[19] G. Huysmans, W. Kerner, D. Borba, H. Holties, and J. Goedbloed, Physics of Plasmas 2, 1605 (1995).

[20] M. Li, B. Breizman, L. Zheng, and E. Y. Chen, New Journal of Physics 17, 125001 (2015).

[21] M. N. Rosenbluth, H. L. Berk, J. W. Van Dam, and D. M. Lindberg, Physics of Fluids B: Plasma Physics 4, 2189 (1992).

[22] F. Zonca and L. Chen, Phys. Rev. Lett. 68, 592 (1992).

[23] S. Poedts, W. Kerner, J. Goedbloed, B. Keegan, G. Huysmans, and E. Schwarz, Plasma physics and controlled fusion 34, 1397 (1992).

[24] M. V. Falessi, N. Carlevaro, V. Fusco, G. Vlad, and F. Zonca, Physics of Plasmas 26, 082502 (2019).

[25] H. Strauss, Journal of Plasma Physics 57, 83 (1997).

[26] M. Hoelzl, P. Merkel, G. Huysmans, E. Nardon, E. Strumberger, R. McAdams, I. Chapman, S. Günter, and K. Lackner, Journal of Physics: Conference Series, 401, 012010 (2012).

[27] H. L. Berk, J. Van Dam, D. Borba, J. Candy, G. Huysmans, and S. Sharapov, Physics of Plasmas 2, 3401 (1995).

[28] G. Halberstadt and J. Goedbloed, Astronomy and Astrophysics 280, 647 (1993). 


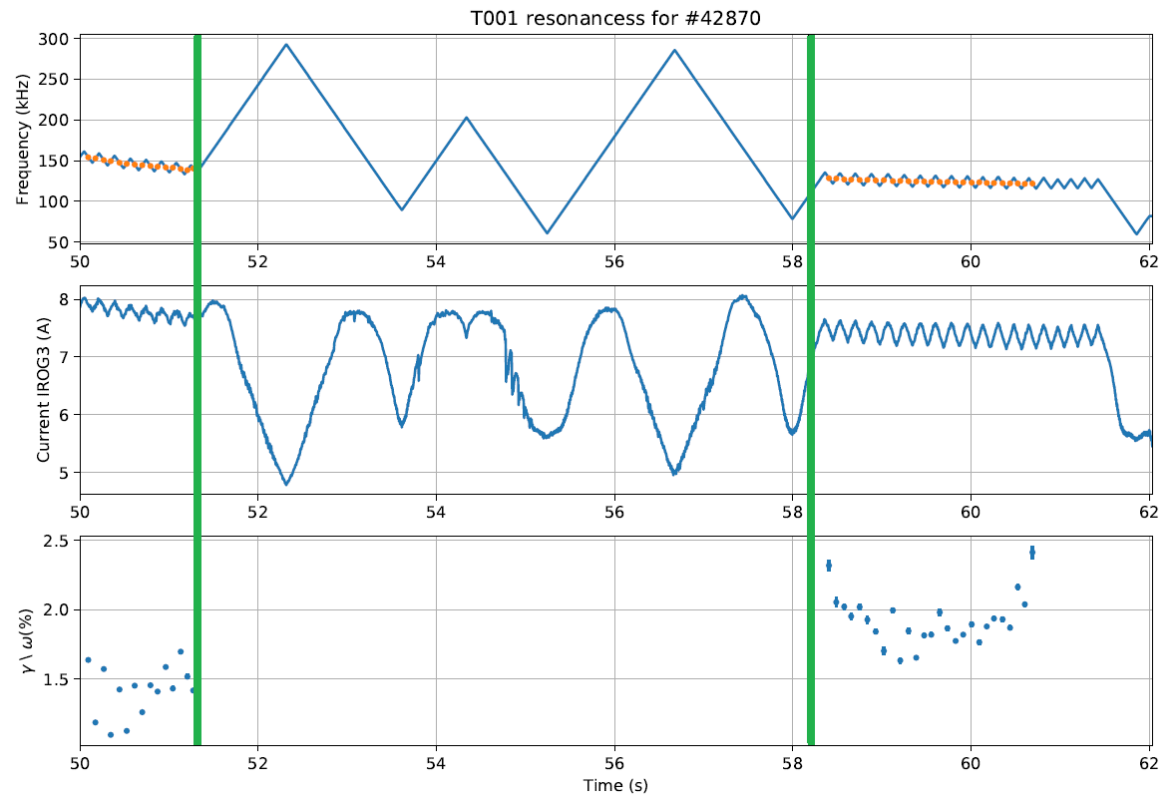

Figure 1: Example of the experimental determination of the TAE resonance in the JET discharge \#42870 with external antenna being used as an active AE diagnostic as explained in [2]. From top to bottom: 1) Blue line: frequency of the externally applied perturbation as a function of time, orange line - tracked TAE frequency as a function of time. 2) Current in the pick-up coils. 3) Relative damping rate of the TAE if it is detected. Note that the time interval $51.4-58.2 \mathrm{~s}$ corresponds to the $X$ point phase of the discharge, with the thich green vertical lines corresponding to the transition moment. The TAE mode could not be tracked in this interval of time. 

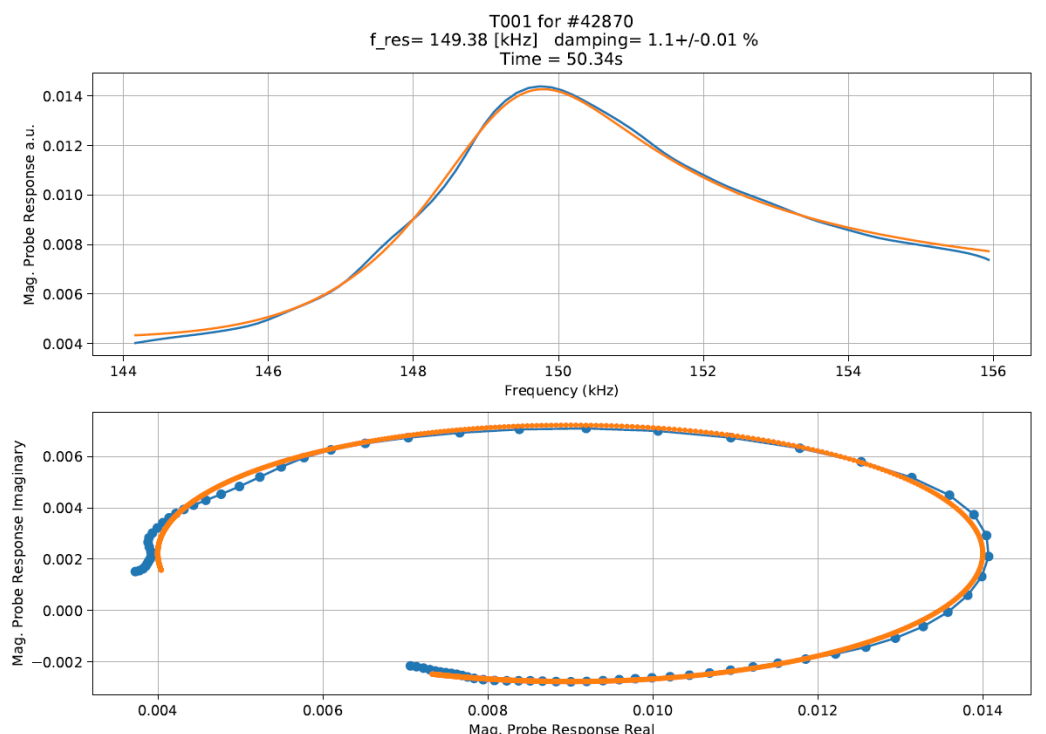

Figure 2: Example of the experimental determination of the TAE resonance in the JET discharge \#42870 based on the technique discussed in [4]. The technique is based on the fitting (solid orange lines) of the transfer function to the experimental magnetic data (blue dotted lines). The absolute value as a function of antenna frequency (top) and the complex plane representation (bottom) of the pick-up coil signal are illustrated. 

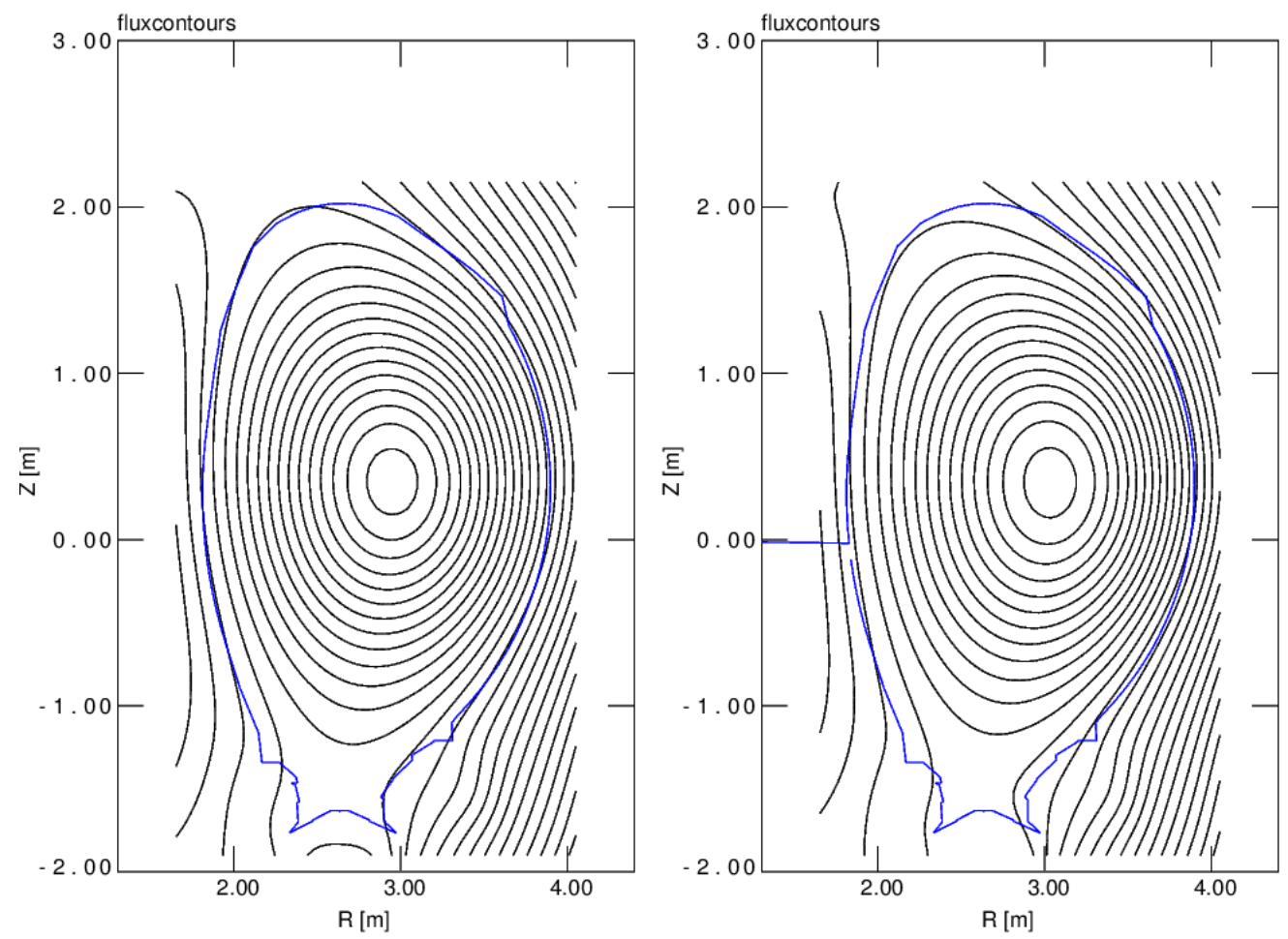

Figure 3: Plasma equilibria reconstructed with the EFIT code for equilibria taken at $t=52.4 \mathrm{~s}$ (left) and $t=54.8 \mathrm{~s}$ (right). Black lines correspond to the flux surfaces, blue line - to the real JET limiter. 


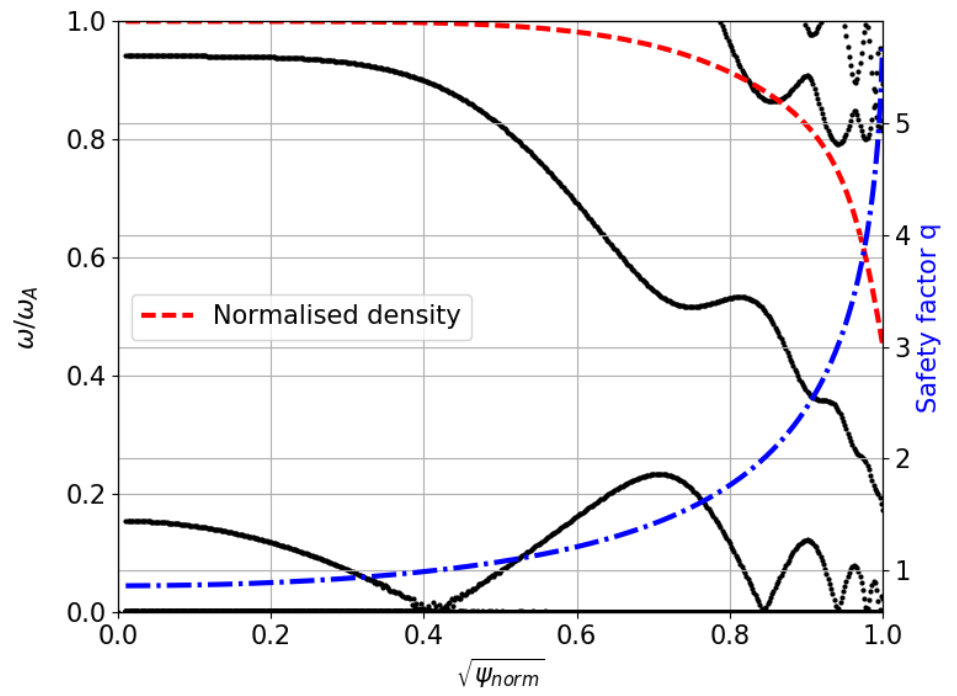

Figure 4: Ideal $n=1$ Alfvén continuum reconstructed with the CSCAS code for the equilibrium taken at $t=54.8 \mathrm{~s}$, together with the corresponding experimental density (red dashed line) and $q$ (blue dash-dotted line) profiles. The experimental density profile was obtained with the LIDAR diagnostics. 


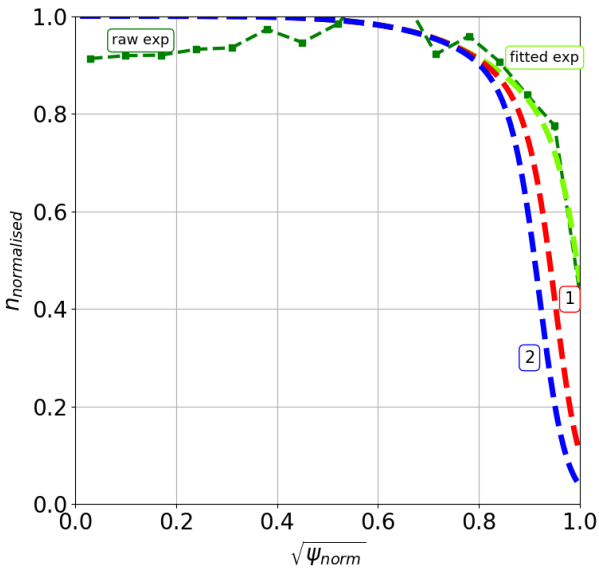

a)

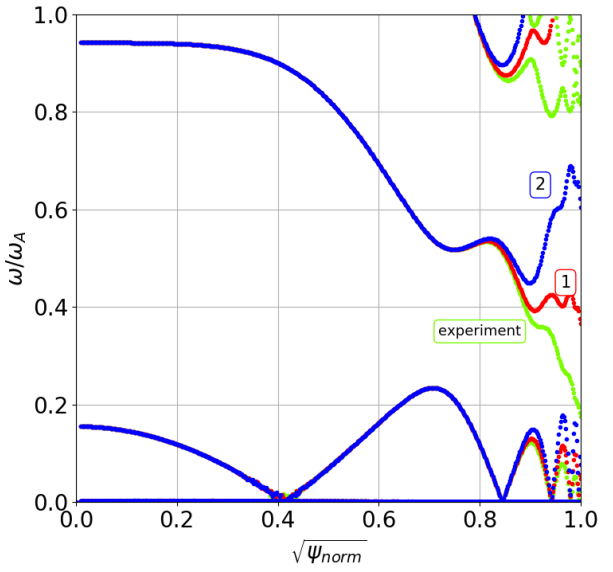

b)

Figure 5: a) Raw and fitted experimental (in green, labeled "raw exp" and "fitted exp") and 2 modified, red (labeled "1") and blue (labeled "2"), density profiles for equilibrium taken at $t=54.8 \mathrm{~s}$. b) Ideal $n=1$ Alfvén continua corresponding to the three different density profiles illustrated in a). It can be seen that the experimental profile corresponds to the continuum with a closed TAE gap, therefore not allowing the TAE identification due to the strong continuum damping. Therefore, the modified density profiles were chosen in such a way that the the TAE is less open (like in the case of the first modified profile), or more open (like in the case of the second modified profile). 


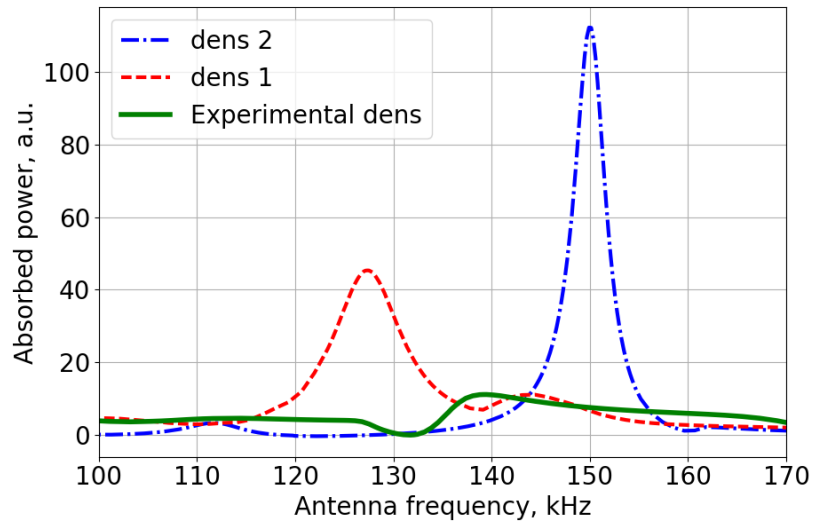

Figure 6: Dependence of the power absorbed by plasma calculated in the CASTOR code as a function of the applied antenna frequency for the three density input profiles shown in fig. 5(a). The response function corresponding to the initial experimental density (in green solid line) shows that the TAE in this case in undetectable. 


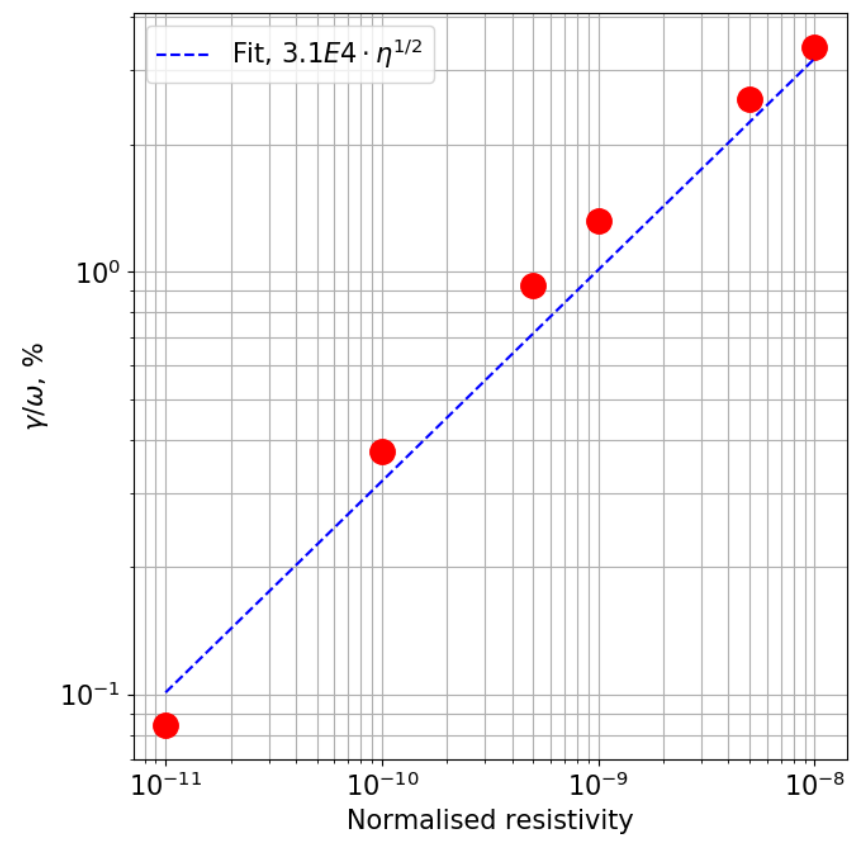

Figure 7: Dependence of the power absorbed by plasma from sweeping antenna signal on the plasma resistivity. The increase of the damping as a function of plasma resistivity suggests the dissipative nature of the TAE damping in the absence of the continuum damping.

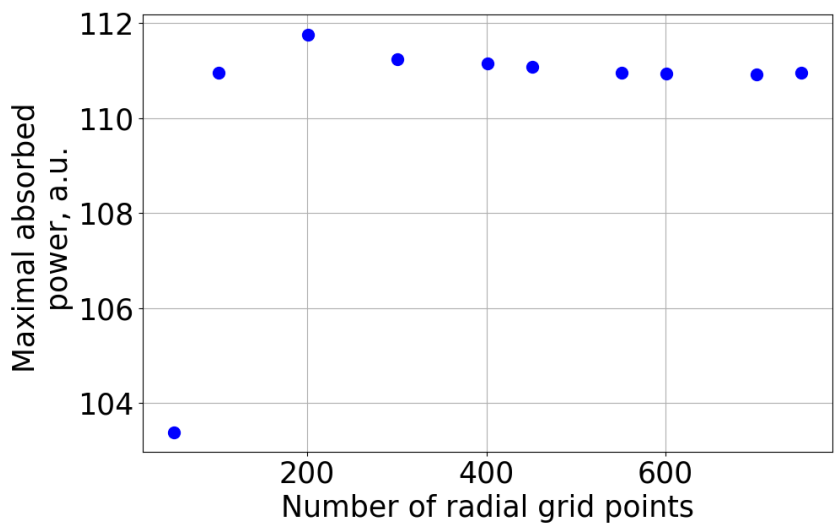

Figure 8: Dependence of the amplitude of the TAE peak on the number of the grid points. The good convergence is achievable from the grid number of 601 points which is used in the simulations. 


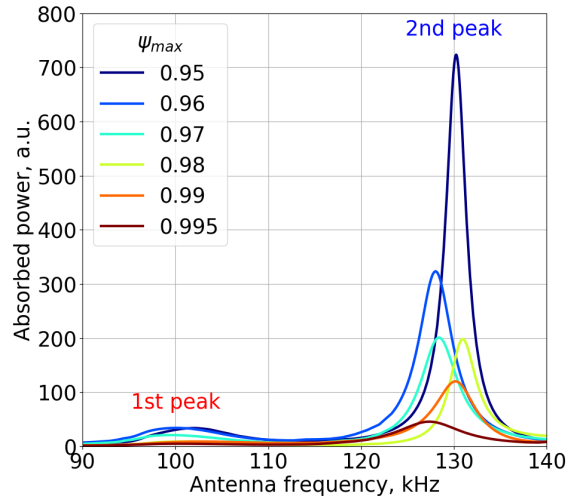

a)

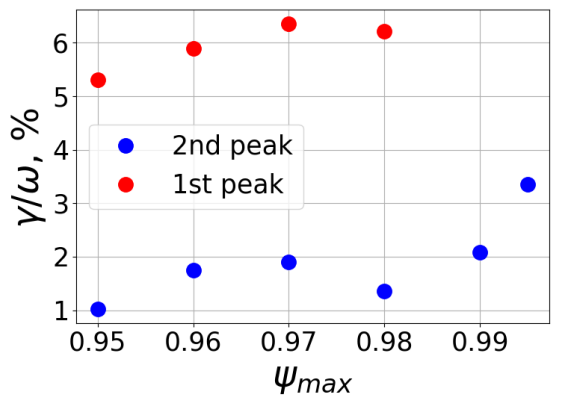

b)

Figure 9: Example of calculation of the a) frequency scan, dependence of the power absorbed by the plasma as a function of the applied antenna signal, and b) the damping rate, determined as HFHM of the TAE response peak. Both are calculated for different normalised poloidal fluxes $\psi_{\max }$ at the boundary of the computational domain. 

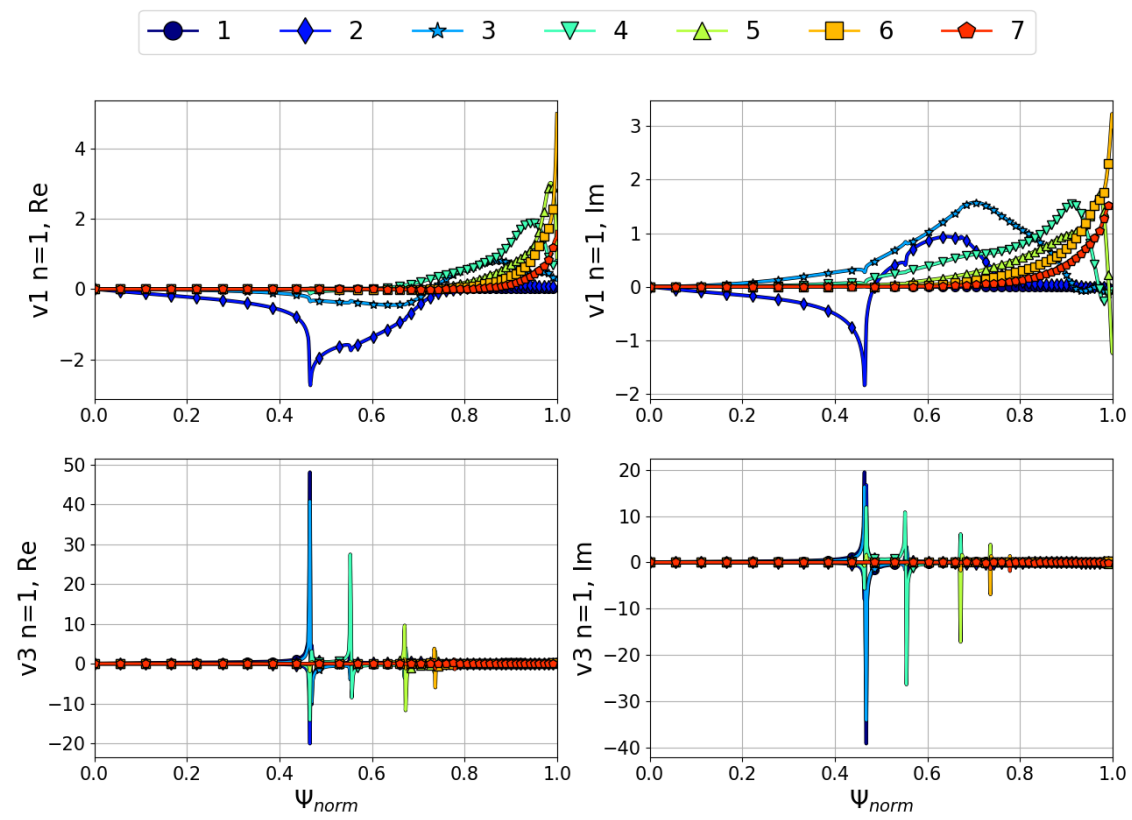

Figure 10: Mode structure of the first $n=1$ TAE peak for the equilibrium at $t=54.8 \mathrm{~s}$ corresponding to the lower resonant frequency. Top row shows the real and imaginary parts of the radial component of the velocity as a function of $s=\sqrt{\psi}$, bottom row - toroidal component of the velocity. Different symbols correspond to the different poloidal harmonics. 

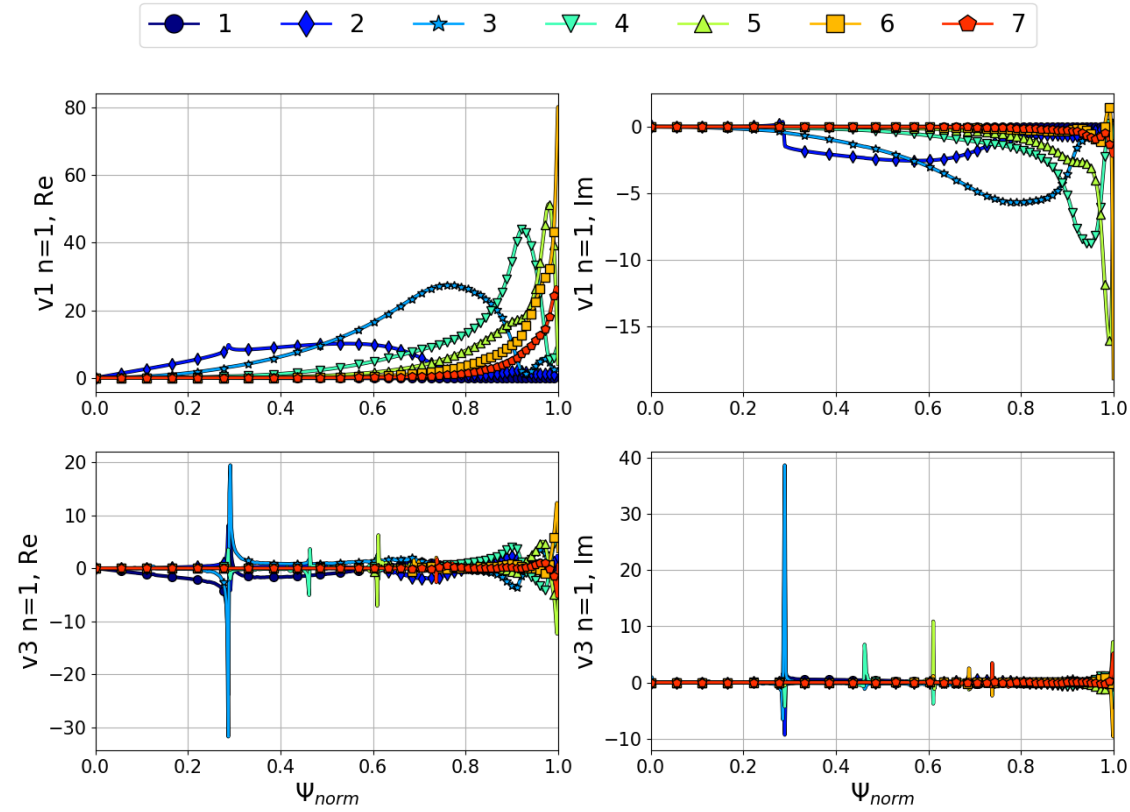

Figure 11: Mode structure of the second $n=1$ TAE peak for the equilibrium at $t=54.8 \mathrm{~s}$ corresponding to the higher resonant frequency. Top row shows the real and imaginary parts of the radial component of the velocity as a function of $s=\sqrt{\psi}$, bottom row - toroidal component of the velocity. Different symbols correspond to the different poloidal harmonics.

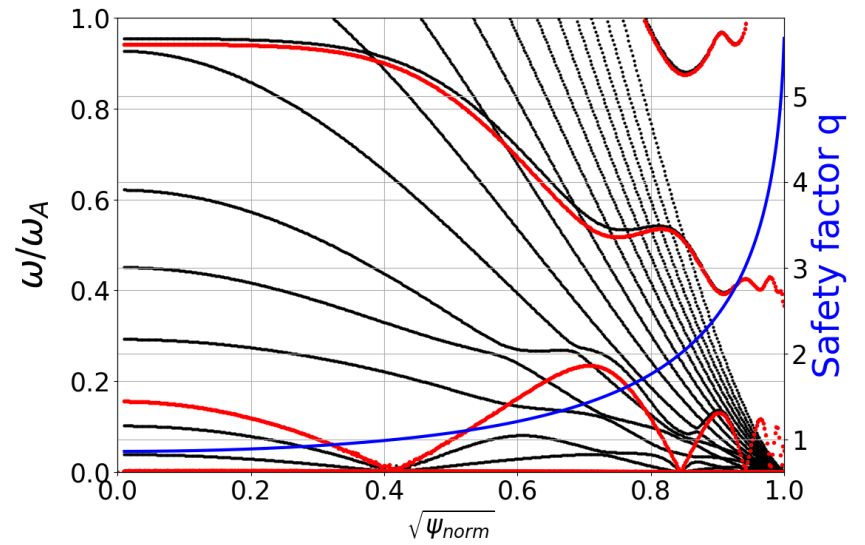

Figure 12: Alfvén continuum with inclusion of the sound waves (in black) and without them (in red). 

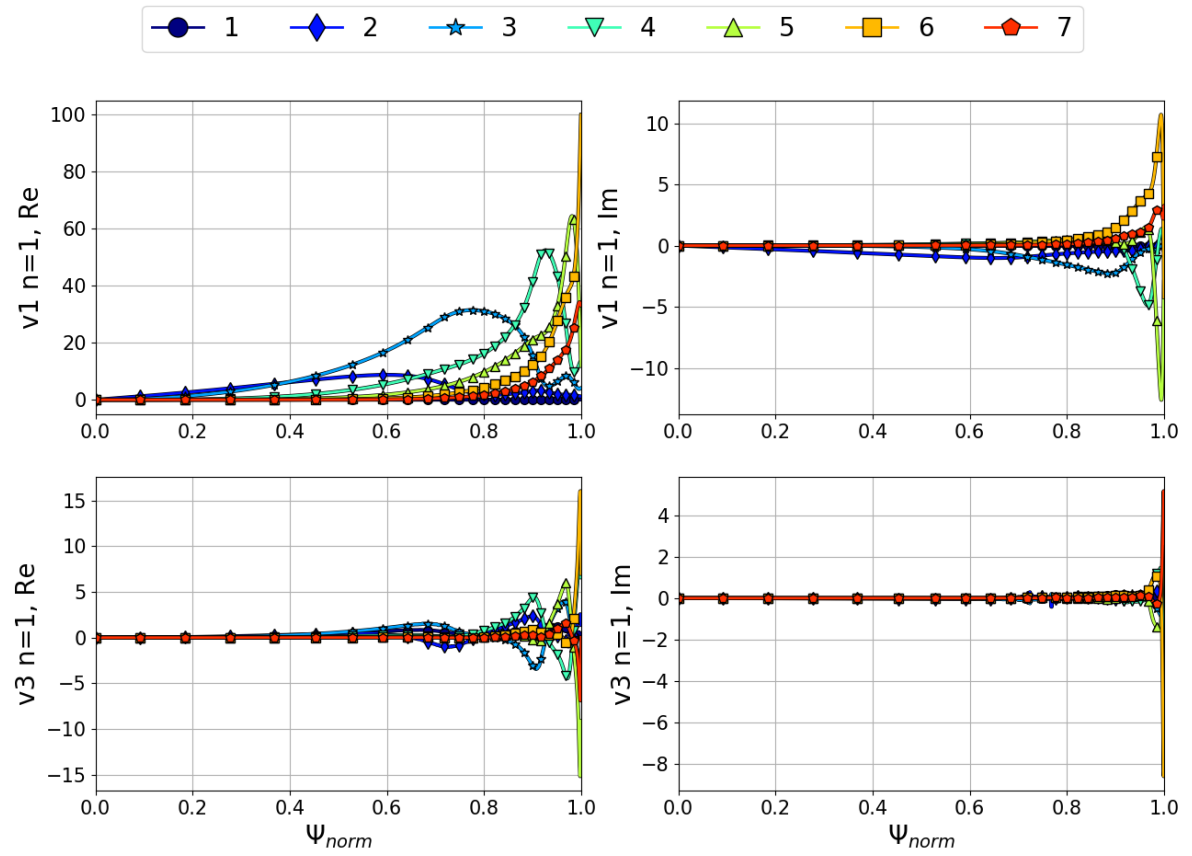

Figure 13: Mode structure of the $n=1$ TAE peak for the equilibrium at $t=54.8 \mathrm{~s}$ without inclusion of the sound waves. Top row shows the real and imaginary parts of the radial component of the velocity as a function of $s=\sqrt{\psi}$, bottom row - toroidal component of the velocity. Different symbols correspond to the different poloidal harmonics. 


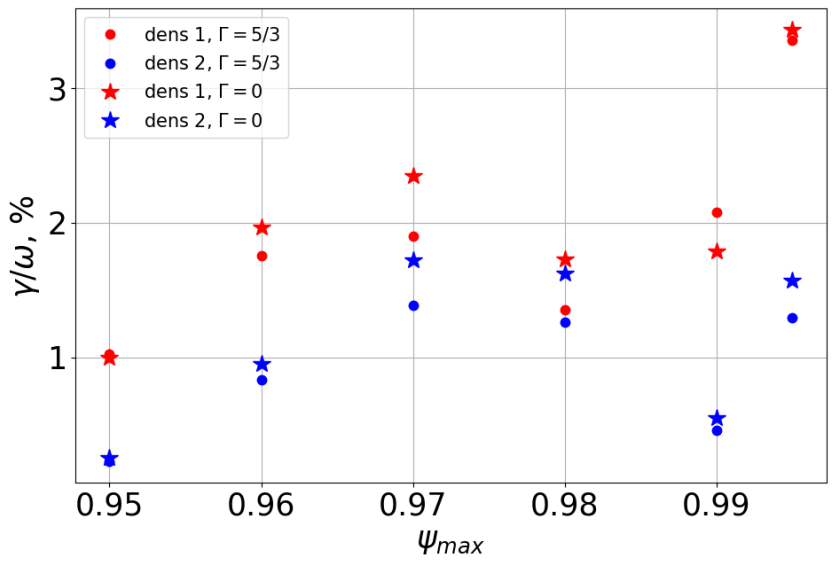

Figure 14: Damping rates as a function of $\psi_{\max }$ for the two modified density profiles as illustrated in fig. 6 and with $(\Gamma=5 / 3)$ or without $(\Gamma=0)$ inclusion of the sound waves.

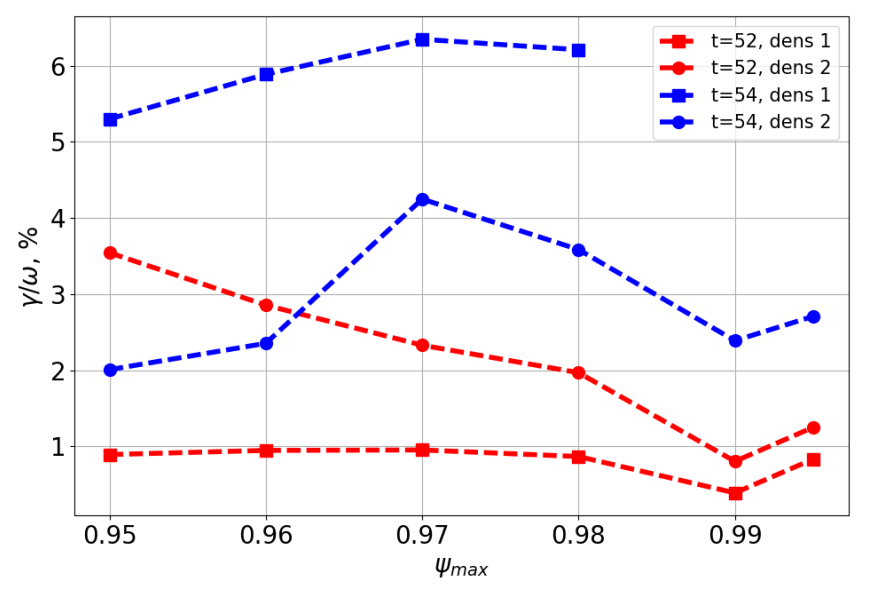

Figure 15: Damping rates as a function of $\psi_{\max }$ for the first TAE peak with the lower frequency. 


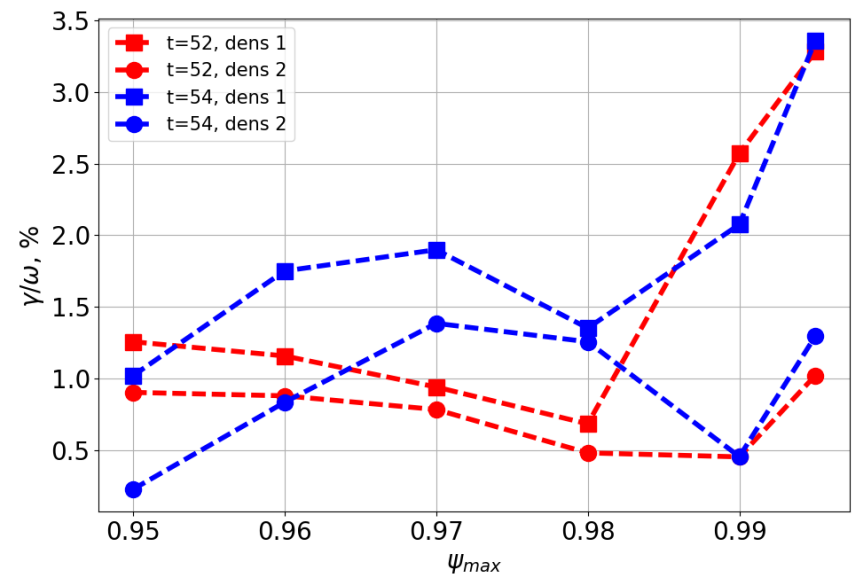

Figure 16: Damping rates as a function of $\psi_{\max }$ for the second TAE peak with the lower frequency.

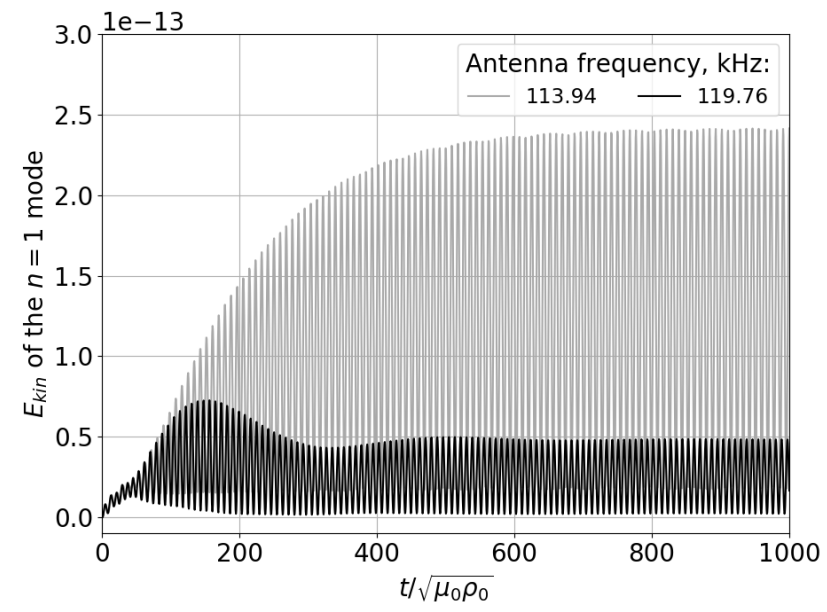

Figure 17: Temporal evolution of the kinetic energy of $n=1$ mode for a resonant case with applied antenna frequency $\omega=114 \mathrm{kHz}$ (in dashed gray) and an off-resonant case with frequency with applied antenna frequency $\omega=120 \mathrm{kHz}$ (in black). 


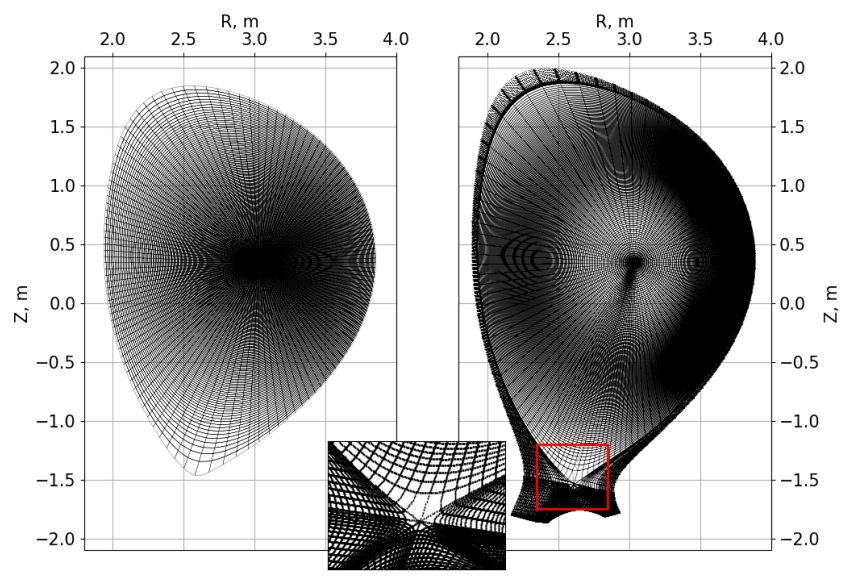

Figure 18: The grids used in the JOREK simulations in the case without the SOL (left) and with the SOL (right).

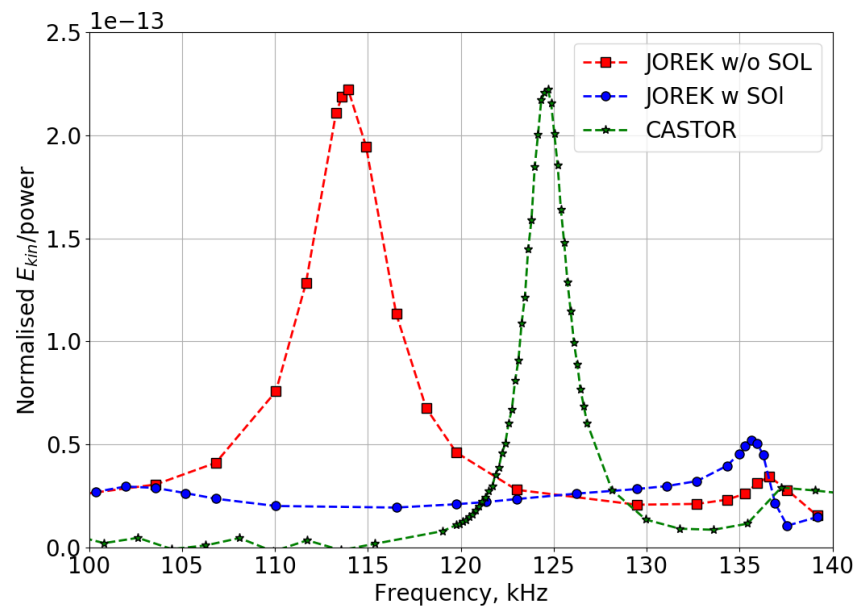

Figure 19: Frequency scan across the TAE gap for an equilibrium at $t=54.8 \mathrm{~s}$. In squares - kinetic energy of the $n=1$ mode in the JOREK simulation without the $S O L$. In circles - kinetic energy of the $n=1$ mode in the JOREK simulation with the SOL. In stars - power absorbed by the plasma calculated via the CASTOR code. Height of the peak is normalised to the peak of the JOREK no-SOL case. 

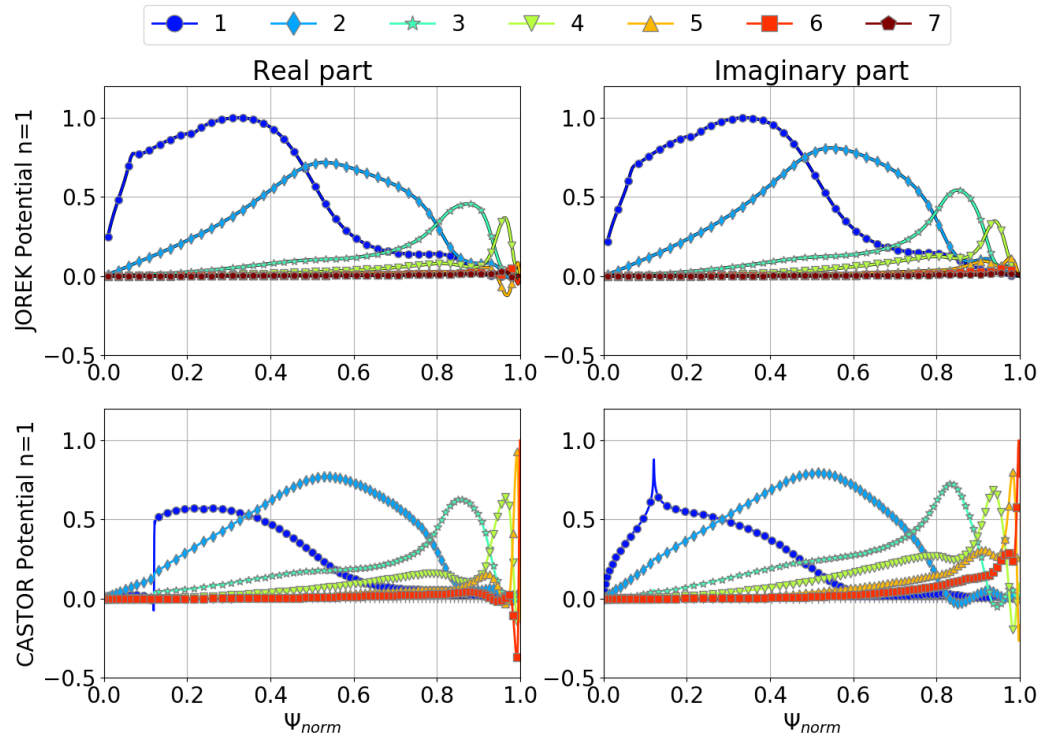

Figure 20: Mode structures of the main TAE peaks in JOREK (top row) and CASTOR (bottom row) simulations. Real and imaginary parts are presented in the left and the right columns respectively. 


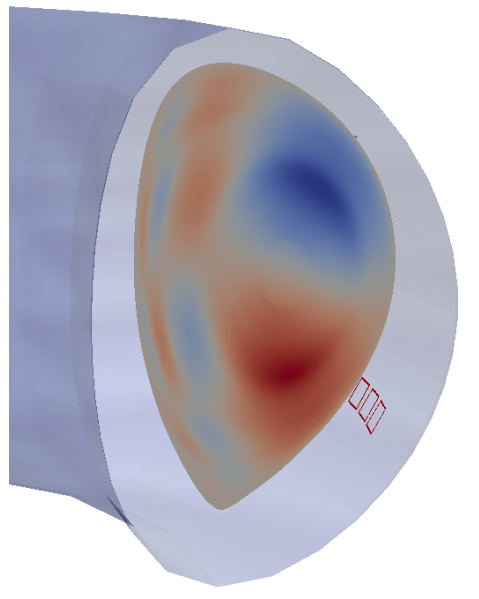

Mode structure of the TAE with lower frequency in the case without inclusion of the SOL.

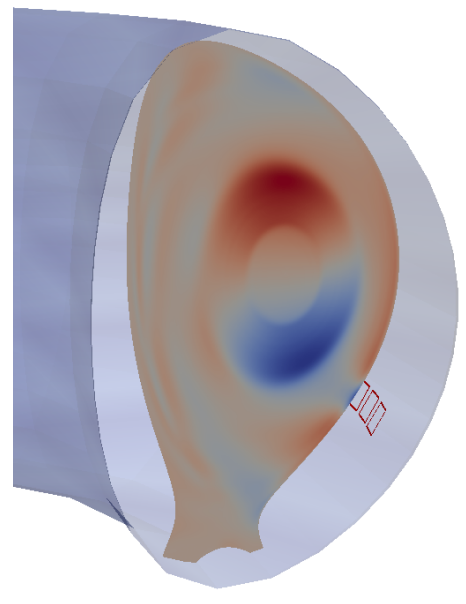

Mode structure of the TAE with lower frequency in the case with inclusion of the SOL.

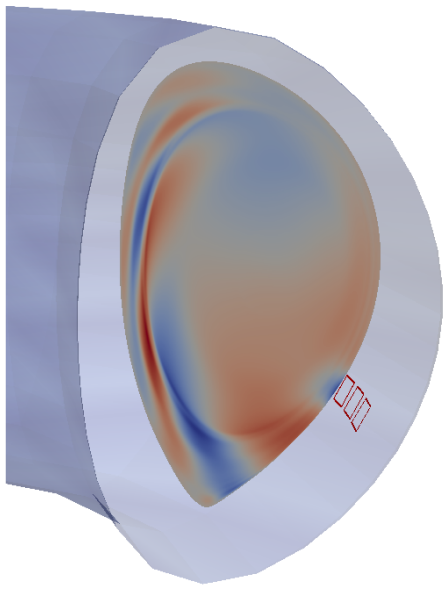

Mode structure of the TAE with higher frequency in the case without inclusion of the SOL.

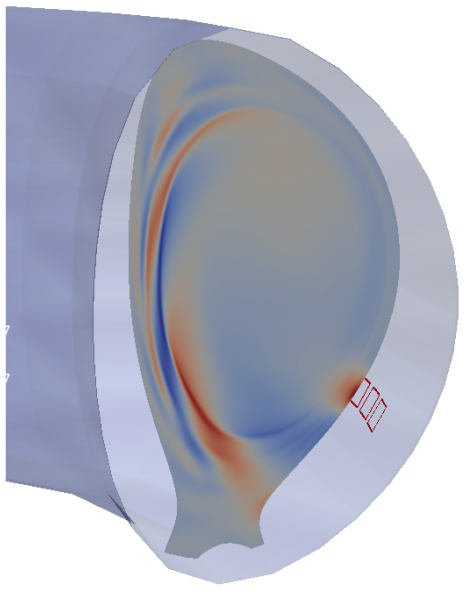

Mode structure of the TAE with higher frequency in the case with inclusion of the SOL.

Figure 21: Poloidal cross-sections representing the mode structure of electric potential of the $n=1$ mode. The mode structures at the top row clearly demonstrate that this an even (left) and odd (right) mode. The red squares visible slightly below the midplane to the right of the main plasma are the antennas used for the TAE excitation. 


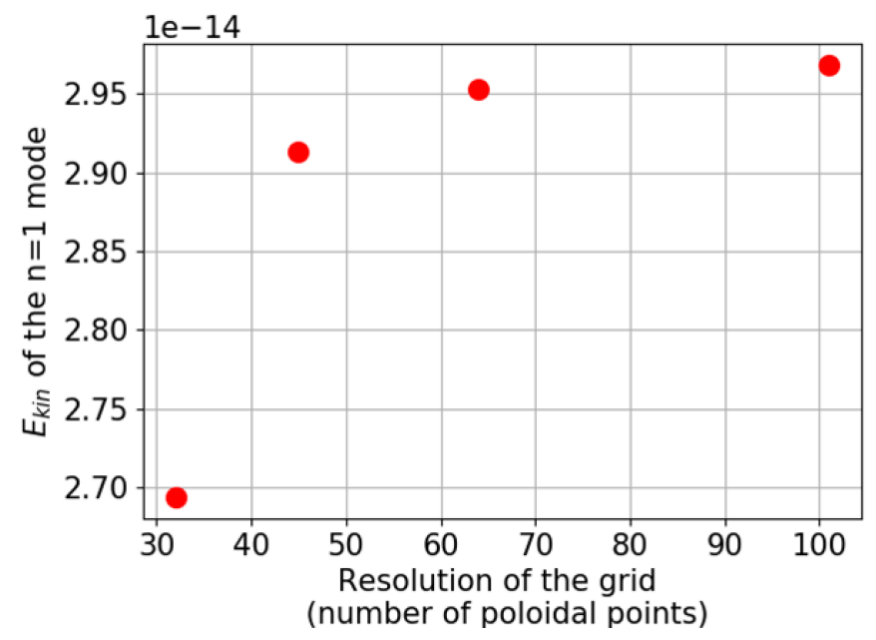

Figure 22: Dependence of the kinetic energy of the $n=1$ mode on the poloidal resolution of the grid.

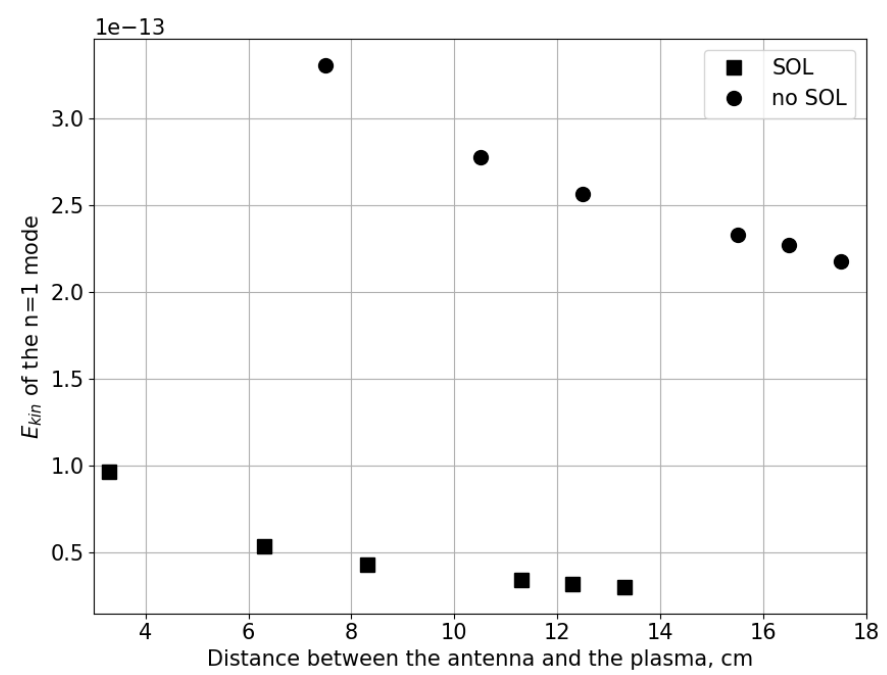

Figure 23: Dependence of the kinetic energy of $n=1$ mode on the distance between antenna and the plasma. 


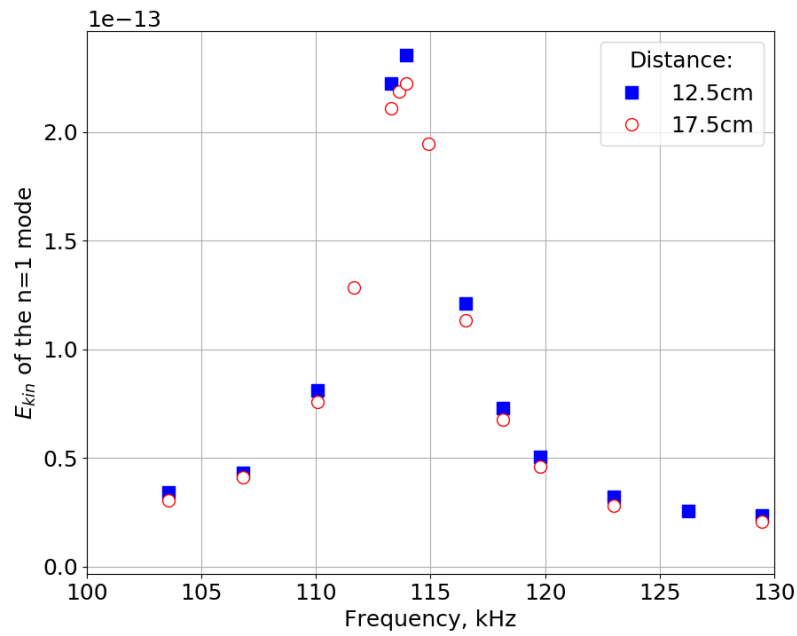

Figure 24: Dependence of the kinetic energy of $n=1$ mode on the applied frequency of the antenna for the no-SOL case with two different antenna positions: $12.5 \mathrm{~cm}$ and $17.5 \mathrm{~cm}$ away from the plasma boundary
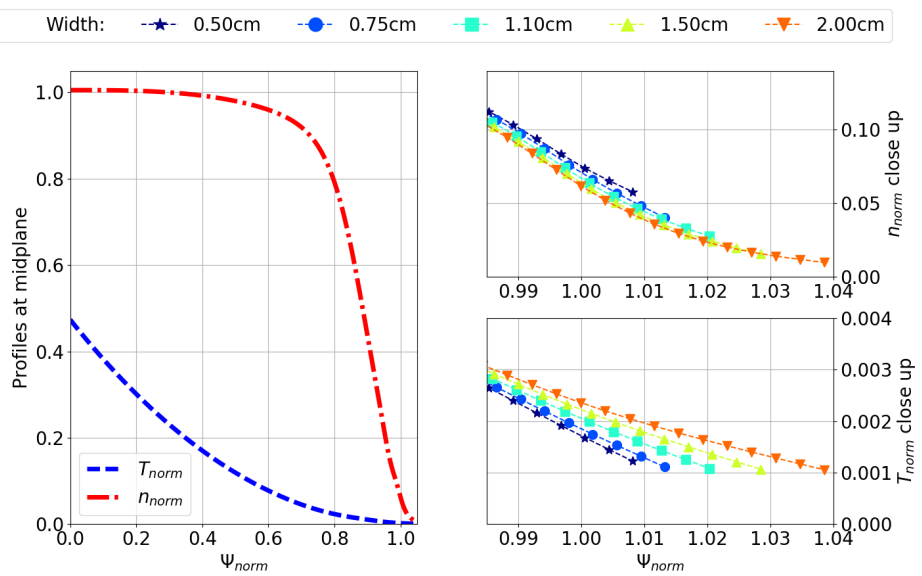

Figure 25: Left: Midplane density (dash-dotted line) and temperature (dashed line) profiles. Right: density (top) and temperature (bottom) profiles in the near-SOL and SOL-region for different midplane SOL widths. 


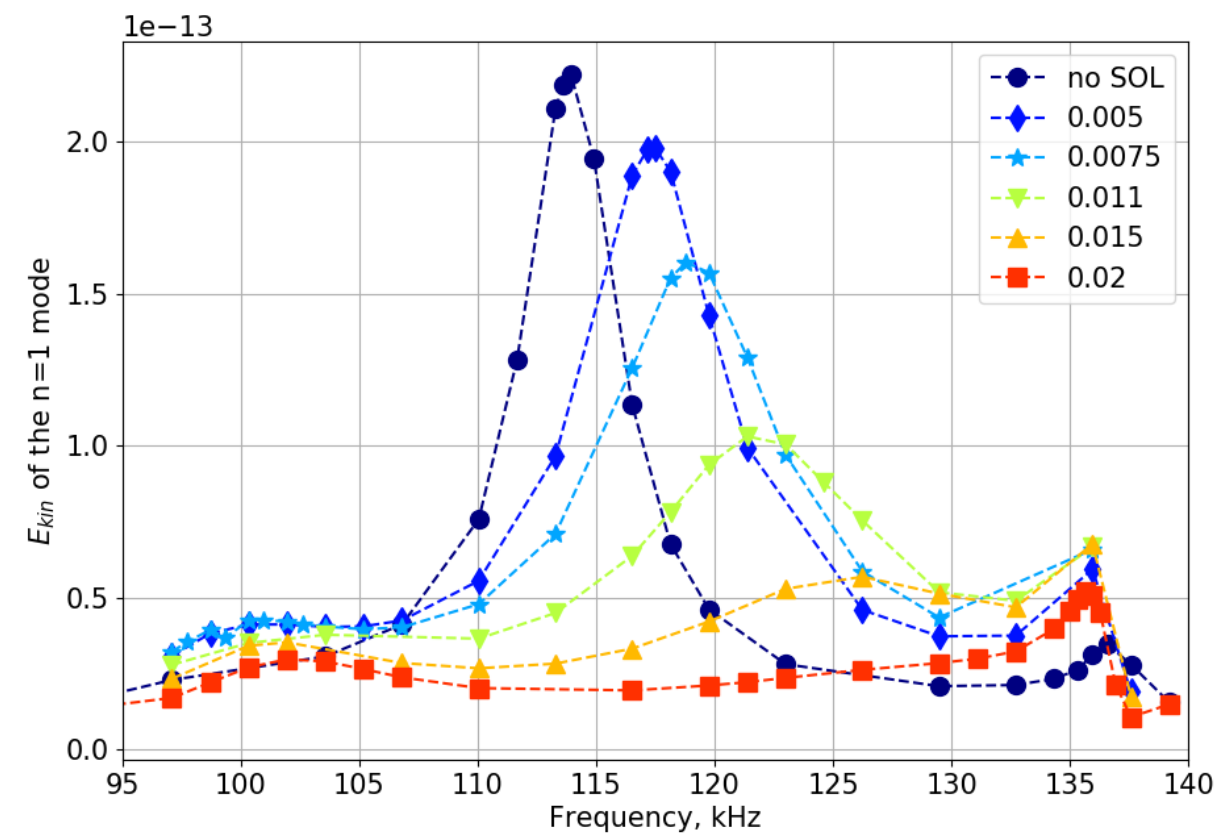

Figure 26: Kinetic energy of the $n=1$ mode as a function of the applied frequency for the different midplane SOL widths. It can be seen in the figure how the main TAE peak located at $f=113 \mathrm{kHz}$ in the no-SOL case gradually decreases with the increase of the SOL width until eventually it cannot be distinguished. 

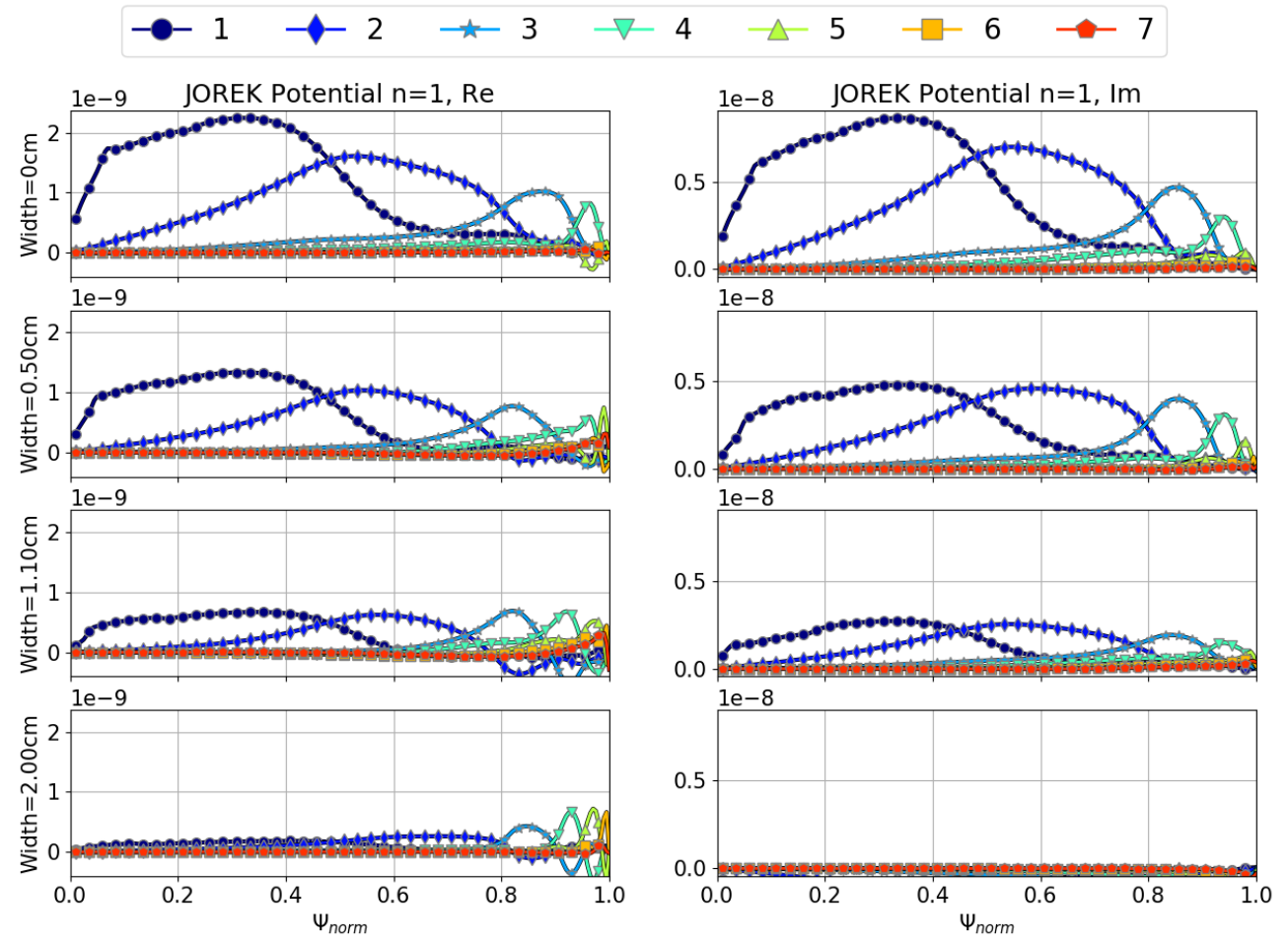

Figure 27: Radial structure of the real (left) and imaginary (right) parts of $n=$ 1 potential of the primary TAE peak for different midplane SOL widths. Different symbols correspond to the different poloidal harmonics. 


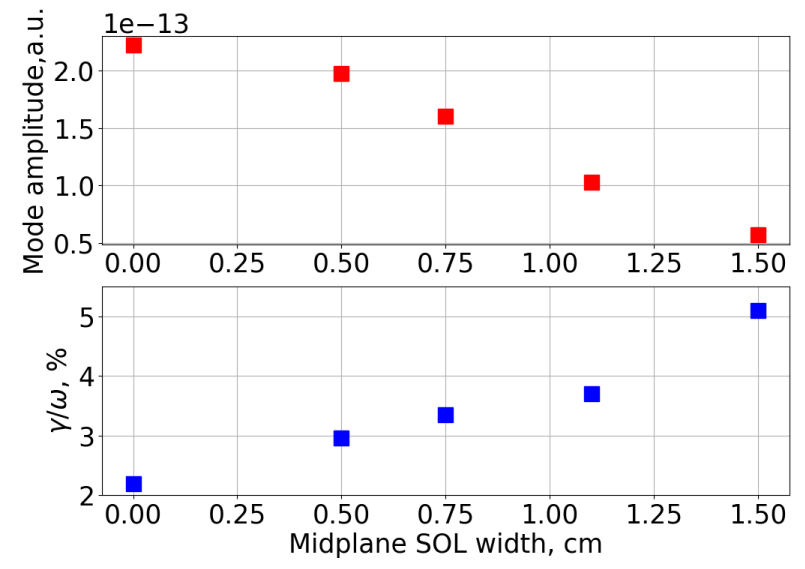

Figure 28: Mode amplitude (top) and damping rate (bottom) as a function of the midplane SOL width. The gradual decrease of the of both the mode amplitude and the damping rate refutes the assumption of the continuum damping on the open field lines as was illustrated for coronal loops in [28]. 